\title{
POSITIVE FRAGMENTS OF RELEVANCE LOGIC AND ALGEBRAS OF BINARY RELATIONS
}

\author{
ROBIN HIRSCH \\ Department of Computer Science, University College London \\ and \\ SZABOLCS MIKULÁS \\ Department of Computer Science and Information Systems, \\ Birkbeck, University of London
}

\begin{abstract}
We prove that algebras of binary relations whose similarity type includes intersection, union, and one of the residuals of relation composition form a nonfinitely axiomatizable quasivariety and that the equational theory is not finitely based. We apply this result to the problem of the completeness of the positive fragment of relevance logic with respect to binary relations.
\end{abstract}

\$1. Introduction. Recently there has been a renewed interest in relation-algebraic semantics for substructural and, in particular, relevance logics (see, e.g., Bimbó et al., 2009; Kowalski, 2007; Maddux, 2007, 2010; Mikulás, 2009, submitted). The idea is roughly the following: formulas are interpreted as binary relations and valid formulas correspond to valid algebraic equations of binary relations. In this paper, we look at the positive fragment $\mathbf{R}_{+}$of relevance logic and the associated semantics by binary relations, and prove a nonfinite axiomatizability result (Theorem 2.2.).

Probably the simplest way of providing an algebraic semantics to (substructural) logics is to consider the Lindenbaum-Tarski algebra of the logic. For instance, the Lambek calculus (LC) is complete w.r.t. ordered residuated semigroups. Similar completeness result holds for relevance logic $\mathbf{R}^{\mathbf{t}}$ w.r.t. De Morgan monoids (cf. Dunn, 1966; Anderson \& Belnap, 1975; Routley \& Meyer, 1973). This type of semantics is rather abstract: the semantics is given by a class of algebras usually defined by a set of axioms.

A more concrete, Kripke-style semantics can be given by evaluating formulas over possible worlds, considering certain connectives as modalities and interpreting them by accessibility relations over the set of possible worlds. For instance, fusion $\circ$ is treated as a binary modality with a corresponding ternary accessibility relation $R$ :

$$
a \models \varphi \circ \psi \Longleftrightarrow \text { there are } b, c \text { such that } R a b c, b \models \varphi \text { and } c \models \psi
$$

compare Meyer \& Routley (1972). Hence we can interpret certain logics via classes of frames of the form $\left(S, R_{i}\right)$, where $S$ is the set of possible worlds and the $R_{i}$ s are accessibility relations over $S$ interpreting the intensional connectives such as fusion or various modalities (in the above example of fusion, $R \subseteq S \times S \times S$ ). Of course, we may have to restrict the class of frames, for example, commutativity of $\circ$ requires: for all $a, b, c$,

$$
R(a, b, c) \Rightarrow R(a, c, b)
$$

Received: February 18, 2010 
and idempotency of $\circ$ requires: for every $a$,

$$
R(a, a, a)
$$

Examples of this type of semantics include the Routley-Meyer semantics for relevance logic $\mathbf{R}$ and its positive fragment $\mathbf{R}_{+}$(Anderson et al., 1972; Routley \& Meyer, 1973) and relational semantics for LC (Došen, 1992; Dunn, 1993). In passing we note that the above algebraic and Kripke-style semantics are not independent: certain axiomatically given classes of algebras can be characterized as (subalgebras of) complex algebras of classes of frames. We mention the classical result of Jónsson \& Tarski (1952) that every relation algebra (see Definition 2.1. below) is a subalgebra of the complex algebra of a frame (or, using the terminology of algebraic logic, of an atom structure). Similar connections via canonical extensions can be established for substructural logics (see, e.g., Gehrke et al., 2005).

Even more concrete semantics can be provided by requiring that the possible worlds have certain properties and the accessibility relations are determined by the nature of the possible worlds. For instance, we may require that possible worlds are ordered pairs, hence formulas are interpreted as binary relations, and the accessibility relation interpreting fusion is

$$
R\left(a_{0}, a_{1}\right)\left(b_{0}, b_{1}\right)\left(c_{0}, c_{1}\right) \Longleftrightarrow a_{0}=b_{0}, a_{1}=c_{1} \text { and } b_{1}=c_{0}
$$

that is, fusion is interpreted as relation composition. This bonds well with the intuition behind dynamic semantics (see, e.g., van Benthem, 1996). In this setting, possible worlds can be thought of as transitions (or information channels) and fusion as dynamic conjunction (the conjuncts are executed/stated sequentially). Implication can be interpreted as the residual $(\mathrm{s})^{1}$ of composition

$$
\left(a_{0}, a_{1}\right) \models \varphi \rightarrow \psi \Longleftrightarrow \text { for every } x,\left(x, a_{0}\right) \models \varphi \text { implies }\left(x, a_{1}\right) \models \psi
$$

and relevant (or De Morgan) negation as converse-complement

$$
\left(a_{0}, a_{1}\right) \models \sim \varphi \Longleftrightarrow\left(a_{1}, a_{0}\right) \forall \varphi
$$

Finite axiomatizability w.r.t. this type of semantics includes the completeness of the relevance logic RM (Maddux, 2010) and the LC (Andréka \& Mikulás, 1994), and the representation of De Morgan lattices (distributive lattices with De Morgan negation) (Dunn, 1982).

Looking at the relevance logic $\mathbf{R}$, propositions are interpreted over a family of commuting and dense binary relations, and the interpretation of the logical connectives is as follows: conjunction $\wedge$ is intersection, disjunction $\vee$ is union, fusion $\circ$ is relation composition, implication $\rightarrow$ is interpreted as the residual of relation composition, and relevant negation $\sim$ as converse-complement. While formulas are evaluated at sets of transitions, truth is restricted to states (or situations), that is, to ordered pairs of the form $(a, a)$ - see the precise definition of this semantics in Section 2.3. Recently Maddux (2007) showed that relevance logic $\mathbf{R}$ indeed can be soundly interpreted over commutative and dense families of relations, and posed the problem of completeness of $\mathbf{R}$ w.r.t. this semantics. It turned out that completeness is impossible in this case (cf. Mikulás, 2009), although completeness can be achieved by adding the mingle axiom to $\mathbf{R}$ and the corresponding requirement that all relations are transitive to the semantics (cf. Maddux, 2010).

1 In the special case where composition is commutative, the right and left residuals of composition coincide, see below. 
As Routley \& Meyer (1973) point out the Routley-Meyer semantics satisfies the variable sharing, or relevance principle: if $\varphi$ and $\psi$ do not share atomic propositions, then $\varphi \rightarrow \psi$ is not valid. Interpreting implication $\rightarrow$ as the residual of fusion avoids some arguably counterintuitive features of classical implication as well. Note that $\left(\varphi_{1} \wedge \varphi_{2}\right) \rightarrow$ $\psi \models\left(\varphi_{1} \rightarrow \psi\right) \vee\left(\varphi_{2} \rightarrow \psi\right)$ is classically valid but does not hold when $\rightarrow$ is interpreted as the residual of fusion. Let $\varphi_{i}$ stand for "you answer Question $i$ " and $\psi$ stand for "you pass the exam," and assume that the rule is that a student who answers both questions passes the exam. We expect that a teacher would have difficulties explaining to two students, one of whom answered Question 1 and the other answered Question 2, why neither of them passed the exam. ${ }^{2}$

Although our results are motivated by substructural logics, they may also be of interest to those studying pure algebraic logic. Recall that a relation algebra $\mathfrak{A}=(A, 0,1, \cdot,+,-, ;$, $\left.\smile, 1^{\prime}\right)$ is a Boolean algebra with operators, and it is representable, an RRA, if it is isomorphic to an algebra of binary relations-see the precise definition in Section 2.2. It is known that RRA is a variety (Tarski, 1955), but it cannot be defined by any finite set of first-order formulas (Monk, 1964), hence the equational theory is not finitely based. There has been some interest in identifying subsignatures $\tau$ of the full relation algebra signature such that the corresponding representation class $Q(\tau)$ (defined formally below) is defined by a finite set of axioms, or where the set of equations valid over $Q(\tau)$ follows from a finite set of equations (see Schein, 1991; Mikulás, 2004; Andréka \& Mikulás, accepted for publication) for surveys. It is known that $\mathrm{Q}(\tau)$ is a finitely axiomatizable quasivariety if $\tau \subseteq\left\{0,1,+, \cdot,-, 1^{\prime},-\right\}$. But the general rule is that $\mathrm{Q}(\tau)$ is not finitely axiomatizable whenever $\in \tau$. A notable exception is $\tau=\{\cdot, ;\}$ (see Bredikhin \& Schein, 1978), but $Q(\tau)$ is known to be not finitely axiomatizable when $\tau$ contains $\{\cdot, \smile, ;\}$ or $\{+, \cdot, ;\}$ (see Hodkinson \& Mikulás, 2000; Andréka, 1991, respectively). Also $Q(\tau)$ is not finitely axiomatizable if $\tau$ equals $\{+, ;\},\left\{\cdot, 1^{\prime}, ;\right\}$, or $\left\{\leq, 1^{\prime}, ;\right\}$ (cf. Andréka, 1988; Hirsch \& Mikulás, 2007; Hirsch, 2005, for respective proofs). If we restrict $\tau$ to positive subsignatures of RRA, and we look at the variety $\mathrm{V}(\tau)$ generated by $\mathrm{Q}(\tau)$, then the picture is more promising: $\mathrm{V}(\tau)$ is finitely based if and only if not all of ;, , and $\smile$ are contained in $\tau$ (see Andréka \& Mikulás, submitted, for this result and for precise references to specific cases).

In this paper we extend these results by looking at generalized subsignatures $\tau$ of RRA, where operations definable in RRA can be included in $\tau$. We show that whenever $\tau$ includes $\cdot,+$, and either the left / or the right residual $\backslash$ of composition, then the quasivariety $\mathrm{Q}(\tau)$ cannot be defined by finitely many formulas, nor can the variety $\mathrm{V}(\tau)$ generated by $\mathrm{Q}(\tau)$ be finitely axiomatized (cf. Theorem 2.3.). Our proof works with the additional requirement of the algebras being dense and commutative, a sufficient condition for soundly interpreting relevance logic $\mathbf{R}$ (see below). Hence we can apply these results to the positive fragment of the relevance logic $\mathbf{R}$ : the relevance logic $\mathbf{R}_{+}$of the language $\{\wedge, \vee, \rightarrow\}$ is not complete w.r.t. algebras of binary relations even if we include finitely many additional axioms to the standard axiomatization of $\mathbf{R}_{+}$(Theorem 2.2.).

The rest of the paper is organized as follows. In the next section, we recall the basics of relevance logic and relation algebras, and state our main results. Section 3 is devoted to the proofs of the key lemmas of the algebraic result. We conclude with some open problems.

2 We heard a similar example from I. Hodkinson, who attributes it to D. Gabbay. 
\$2. Relevance logic and relation algebras. We continue with recalling the basics of relevance logic.

2.1. Relevance logic. We recall that the logic $\mathbf{R}$ of relevant implication is a finite Hilbert-style derivation system in the language $\mathcal{L}(\mathbf{R})=\{\rightarrow, \wedge, \sim\}$ (see, e.g., Routley $\&$ Meyer, 1973; Anderson \& Belnap, 1975; Anderson et al., 1992). In $\mathbf{R}$ the connectives $\vee$ and $\circ$ are defined as

$$
\varphi \vee \psi=\sim(\sim \varphi \wedge \sim \psi) \text { and } \varphi \circ \psi=\sim(\varphi \rightarrow \sim \psi)
$$

In Anderson \& Belnap (1975), $\mathbf{R}$ is defined by the following axioms

$$
\begin{aligned}
A 1 & \varphi \rightarrow \varphi \\
A 2 & \varphi \rightarrow((\varphi \rightarrow \psi) \rightarrow \psi) \\
A 3 & (\varphi \rightarrow \psi) \rightarrow((\psi \rightarrow \eta) \rightarrow(\varphi \rightarrow \eta)) \\
A 4 & (\varphi \rightarrow(\varphi \rightarrow \psi)) \rightarrow(\varphi \rightarrow \psi) \\
A 5 & (\varphi \wedge \psi) \rightarrow \varphi \\
A 6 & (\varphi \wedge \psi) \rightarrow \psi \\
A 7 & ((\varphi \rightarrow \psi) \wedge(\varphi \rightarrow \eta)) \rightarrow(\varphi \rightarrow(\psi \wedge \eta)) \\
A 8 & \varphi \rightarrow(\varphi \vee \psi) \\
A 9 & \psi \rightarrow(\varphi \vee \psi) \\
A 10 & ((\varphi \rightarrow \eta) \wedge(\psi \rightarrow \eta)) \rightarrow((\varphi \vee \psi) \rightarrow \eta)) \\
A 11 & (\varphi \wedge(\psi \vee \eta)) \rightarrow((\varphi \wedge \psi) \vee \eta) \\
A 12 & (\varphi \rightarrow \sim \psi) \rightarrow(\psi \rightarrow \sim \varphi) \\
A 13 & \sim \sim \varphi \rightarrow \varphi
\end{aligned}
$$

and inference rules

$$
\begin{array}{ll}
R 1 & \varphi, \varphi \rightarrow \psi \vdash \psi \\
R 2 & \varphi, \psi \vdash \varphi \wedge \psi
\end{array}
$$

We will write $\mathbf{R} \vdash \varphi$ if $\varphi$ is derivable from the axioms, that is, if $\varphi$ is a theorem of $\mathbf{R}$.

The derivation system $\mathbf{R M}$ has the additional mingle axiom

$$
\varphi \rightarrow(\varphi \rightarrow \varphi)
$$

The logic $\mathbf{R}^{\mathbf{t}}$ is defined by expanding the language of $\mathbf{R}$ by a logical constant $\mathbf{t}: \mathcal{L}\left(\mathbf{R}^{\mathbf{t}}\right)=$ $\mathcal{L}(\mathbf{R}) \cup\{\mathbf{t}\}$, and adding the following two axioms to those of $\mathbf{R}$ :

$$
\mathbf{t} \quad \text { and } \quad \mathbf{t} \rightarrow(\varphi \rightarrow \varphi)
$$

The positive fragment $\mathbf{R}_{+}$of $\mathbf{R}$ is defined by the axioms $A 1-A 11$ and the derivation rules $R 1$ and $R 2$. For more details on relevance logic we refer the reader to the monographs (Anderson \& Belnap, 1975; Anderson et al., 1992).

Maddux (2007) observes that a sound semantics is provided for relevance logic by algebras of binary relations. Next we recall the definition of representable relation algebras and then describe how to interpret relevance logic.

2.2. Relation algebras. Let us recall the basic definitions about relation algebras (cf. Hirsch \& Hodkinson, 2002; Maddux, 2006). 


\section{DEFINITION 2.1.}

1. A relation algebra, an $\mathrm{RA}$, is an algebra

$$
\mathfrak{A}=\left(A, 0,1, \cdot,+,-, ;, 1^{\prime}\right)
$$

such that $(A, 0,1, \cdot,+,-)$ is a Boolean algebra, and the following equations hold, for every $x, y, z \in A$ :

$$
\begin{array}{ll}
(R 1) & x ;(y ; z)=(x ; y) ; z \\
(R 2) & (x+y) ; z=(x ; z)+(y ; z) \\
(R 3) & x ; 1^{\prime}=x \\
(R 4) & x \smile \smile=x \\
(R 5) & (x+y) \smile=x \smile+y^{\smile} \\
(R 6) & (x ; y) \smile=y \smile x \\
(R 7) & x^{\smile} ;(-(x ; y)) \leq-y
\end{array}
$$

where $x \leq y$ abbreviates $x+y=y$. We denote the class of all relation algebras by $\mathrm{RA}$. We call a relation algebra $\mathfrak{A}$ integral if $1^{\prime}$ is an atom (minimal nonzero element) in $\mathfrak{A}$, and symmetric if every element is self-converse $\left(x=x^{\smile}\right)$.

2. By a proper relation algebra, a PRA, we mean an algebra $\mathfrak{A}=(A, 0,1, \cdot,+,-$, $\left.;, \smile, 1^{\prime}\right)$ such that $A \subseteq \mathcal{P}(W)$ (the power set of $W$ ) for some equivalence relation $W, 0=\emptyset, 1=W, \cdot$ is intersection, + is union, - is complement w.r.t. $W$, ; is relation composition, $\smile$ is relation converse, and $1^{\prime}$ is the identity relation ${ }^{\prime} \mathrm{d}_{W}$ restricted to $W$. More formally, for all elements $x, y \in A$,

$$
\begin{aligned}
x ; y & =\{(u, v) \in W:(u, w) \in x \text { and }(w, v) \in y \text { for some } w\} \\
x^{\smile} & =\{(u, v) \in W:(v, u) \in x\} \\
1^{\prime} & =\{(u, v) \in W: u=v\}
\end{aligned}
$$

We denote the class of proper relation algebras by PRA. Given an $\mathfrak{A} \in \mathrm{PRA}$ and $W$ as above, we call $W$ the unit of $\mathfrak{A}$. By a relation set algebra, an $\mathrm{Rs}$, we mean a proper relation algebra $\mathfrak{A}$ with a square unit: $W=U \times U$ for some set $U$, the base of $\mathfrak{A}$. We denote the class of relation set algebras by $\mathrm{Rs}$.

The class RRA of representable relation algebras is defined as

$$
\mathrm{RRA}=\mathrm{IPRA}
$$

that is, we close the class PRA under isomorphic copies.

Note that the (quasi)equational theories of Rs, PRA, and RRA coincide. In fact we have

$$
\text { RRA }=\text { IPRA }=\text { SPRS }
$$

that is, representable algebras are given by the closure of the class Rs under products and (isomorphic copies of) subalgebras. Indeed, if we take an arbitrary $\mathfrak{A} \subseteq \prod_{i \in I} \mathfrak{A}_{i}$ where each $\mathfrak{A}_{i} \in \mathrm{Rs}$, then $\mathfrak{A}$ may be embedded into the full algebra on $\mathcal{P}(W)$, where $W$ is the disjoint union of the units $W_{i}$ of $\mathfrak{A}_{i}$, that is, $\mathfrak{A} \in \mathrm{RRA}$. Conversely, an $\mathfrak{A} \in$ PRA with an equivalence relation unit $W$ is a subalgebra of the product of the restrictions $\mathfrak{A}_{i}$ of $\mathfrak{A}$ to the equivalence classes $W_{i}$ of $W$, whence $\mathfrak{A} \in \mathbf{S P R s}$ (see Hirsch \& Hodkinson, 2002, Lemma 3.7.).

We may introduce additional operations in addition to, or instead of, the operations for $\mathrm{RA}$ as follows. An RA-definable operation is a term $t(\bar{x})$ using only the constants and the 
operations in $\left\{0,1,+, \cdot,-, 1^{\prime}, \smile, ;\right\}$ and the variables in $\bar{x}$ (a sequence of variables). The main additional operations considered in this paper are the two residuals of composition, $\backslash$ and /

$$
x \backslash y=-\left(x^{\smile} ;-y\right) \text { and } x / y=-\left(-x ; y^{\smile}\right)
$$

It is easy to check that in any relation algebra $\mathfrak{A}$, and for any $x, y, z \in A$,

$$
y \leq x \backslash z \text { iff } x ; y \leq z \text { iff } x \leq z / y
$$

We note that for any $x_{1}, x_{2}, y \in A$,

$$
\begin{aligned}
& \left(x_{1} \backslash y\right) \cdot\left(x_{2} \backslash y\right)=\left(x_{1}+x_{2}\right) \backslash y \\
& \left(y \backslash x_{1}\right) \cdot\left(y \backslash x_{2}\right)=y \backslash\left(x_{1} \cdot x_{2}\right)
\end{aligned}
$$

with similar equations holding for the left residual. Thus each residual is monotonic in one argument and reverse monotonic (or antitonic, or downward monotonic) in the other argument. The interpretation of the residuals in a PRA with unit $W$ is given by

$$
\begin{aligned}
& x \backslash y=\{(u, v) \in W: \text { for every } w,(w, u) \in x \text { implies }(w, v) \in y\} \\
& x / y=\{(u, v) \in W: \text { for every } w,(v, w) \in y \text { implies }(u, w) \in x\}
\end{aligned}
$$

see Figure 1. We also note that converse-complement $\sim x$ is defined as $\sim x=-\left(x^{\smile}\right)$ in $\mathrm{RA}$, and it is interpreted in a PRA with unit $W$ as

$$
\sim x=\{(u, v) \in W:(v, u) \notin x\}
$$

Let $\tau$ be a subset of the definable operations in RA. We will denote the class of subalgebras of the $\tau$-reducts, the $\tau$-subreducts, of the elements of Rs by $\mathrm{R}(\tau)$.

Next we define subclasses of $\mathrm{R}(\tau)$ satisfying some extra conditions. We call an algebra $\mathfrak{A}$ in $\mathrm{R}(\tau)$ commutative if, for every $x, y \in A$ and $u, v, w$,

$$
(u, w) \in x \text { and }(w, v) \in y \text { imply }\left(u, w^{\prime}\right) \in y \text { and }\left(w^{\prime}, v\right) \in x \text { for some } w^{\prime}
$$

and dense if, for every $x \in A$ and $u, v$,

$$
(u, v) \in x \text { implies }(u, w) \in x \text { and }(w, v) \in x \text { for some } w
$$

compare the similar "frame conditions" (2) and (3) on Routley-Meyer frames (Routley \& Meyer, 1973). Let $\mathbf{R}^{c d}(\tau)$ denote the subclass of $\mathbf{R}(\tau)$ where each algebra is commutative and dense. If composition ; is definable in $\mathrm{R}(\tau)$, then commutativity and density are expressible by the equations $x ; y=y ; x$ and $x \leq x ; x$, respectively. It is easy to check that $x \backslash y$ and $y / x$ coincide in commutative algebras, hence in $\mathrm{R}^{c d}(+, \cdot, \backslash)$.

Let $\mathrm{Q}(\tau)$ and $\mathrm{Q}^{c d}(\tau)$ denote the quasivarieties generated by $\mathrm{R}(\tau)$ and $\mathrm{R}^{c d}(\tau)$, respectively, and let $\mathrm{V}(\tau)$ and $\mathrm{V}^{c d}(\tau)$ denote the corresponding varieties. In general, $\mathrm{Q}(\tau)$ and
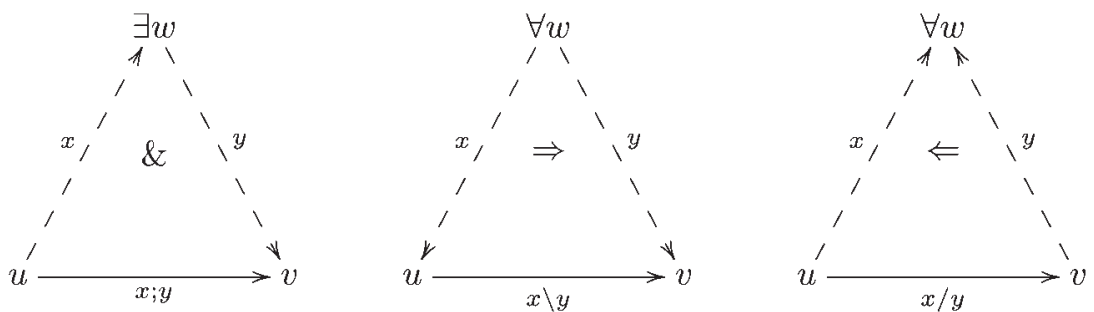

Fig. 1. Composition and its residuals. 
$\mathrm{Q}^{c d}(\tau)$ are not closed under homomorphic images, whence the generated varieties $\mathrm{V}(\tau)$ and $\mathrm{V}^{c d}(\tau)$ might be strictly bigger than these quasivarieties (in contrast to the case of the full RA signature). In passing we note that $\mathrm{R}(\tau)$ is closed under ultraproducts (but may not be closed under products), whence $\mathrm{Q}(\tau)=\operatorname{SPR}(\tau)$, that is, elements of the generated quasivariety are representable as algebras of binary relations just like in the case of the full RA signature.

2.3. Main results. Relevance logic $\mathbf{R}$ can be interpreted into $R^{c d}(\cdot, \sim, \backslash)$ as follows. Let $\mathfrak{A} \in \mathrm{R}^{c d}(\cdot, \sim, \backslash)$ with unit $W$. A valuation is a map $v$ from the set of propositional atoms into $A$ that is extended to compound formulas by interpreting the logical connectives $\wedge, \rightarrow$, and $\sim$ by the corresponding algebraic operations $\cdot, \backslash$, and $\sim$, respectively. We define

$$
\operatorname{Id}_{W}=\{(u, v) \in W: u=v\}
$$

Note that $a \backslash a \supseteq \operatorname{Id}_{W}$ for any $a \in A$, but $\operatorname{ld}_{W}$ may not be an element of $\mathfrak{A}$. Nevertheless, it can be used to define the semantics of $\mathbf{R}$ in $\mathfrak{A}$ as follows:

$$
\mathfrak{A} \models \varphi \text { iff } \operatorname{Id}_{W} \subseteq v(\varphi) \text { for every valuation } v .
$$

The formula $\varphi$ is valid in $\mathrm{R}^{c d}(\cdot, \sim, \backslash)$, in symbols $\mathrm{R}^{c d}(\cdot, \sim, \backslash) \models \varphi$, iff $\mathfrak{A} \models \varphi$ for every $\mathfrak{A} \in \mathrm{R}^{c d}(\cdot, \sim, \backslash)$. As Maddux (2007) shows this is a sound semantics for $\mathbf{R}$ :

$$
\mathbf{R} \vdash \varphi \text { implies } \mathbf{R}^{c d}(\cdot, \sim, \backslash) \models \varphi
$$

In Mikulás (2009) it is shown that completeness does not hold. In passing we note that RM is complete and sound w.r.t. $\mathbf{R}^{c d t}(\cdot, \sim, \backslash)$, the subclass of $\mathbf{R}^{c d}(\tau)$ defined by the additional requirement of transitivity: $x ; x \leq x$ (Maddux, 2010).

The proof in Mikulás (2009) heavily uses the existence of the connective $\sim$. Thus it leaves the problem whether $\mathbf{R}_{+}$is complete w.r.t. $\mathbf{R}^{c d}(\cdot,+, \backslash)$ open (of course, $\vee$ is interpreted as + ). A related question is whether we can finitely axiomatize the logic of $\mathrm{R}^{c d}(\cdot,+, \backslash, ;)$ (where ; is the interpretation of fusion $\circ$ ). Here we show that no completeness is possible in these cases.

THEOREM 2.2. Relevance logic $\mathbf{R}_{+}$is not complete w.r.t. to the semantics $\mathbf{R}^{c d}(\cdot,+, \backslash)$. In fact, the logic of $\mathrm{R}^{c d}(\cdot,+, \backslash)$ is not axiomatizable by a finite set of axioms and the derivation rules $R 1$ and $R 2$. The same nonfinite axiomatizability holds for $\mathrm{R}^{c d}(\tau)$ and $\mathrm{R}(\tau)$, where $\{\cdot,+, \backslash\} \subseteq \tau$ and the elements of $\tau$ are definable using $\cdot,+, \backslash, /, \sim$, and $1^{\prime}$.

Proof. Observe that an equation $\sigma \leq \tau$ is valid in $\mathfrak{A} \in \mathrm{R}^{c d}(\cdot,+, \backslash)$ if and only if $\operatorname{ld}_{W} \subseteq \sigma \backslash \tau$ in $\mathfrak{A}$. Thus the theorem is an immediate consequence of Theorem 2.3. below.

We formulate the main algebraic result of the paper.

THEOREM 2.3. Let $\tau$ be a similarity type, definable by the operations of relation algebra, such that $+, \cdot, \backslash$ are definable by $\tau$. Then the equational and quasiequational theories of $\mathrm{R}(\tau)$ and $\mathrm{R}^{c d}(\tau)$ are not finitely based.

Symmetrically, we may prove the same results if $\tau$ defines $+, \cdot, /$.

Proof. For every $n \in \omega$ we will define commutative and dense $\mathfrak{A}_{n} \in \mathrm{RA}$ and show that

1. for every $2 \leq n \in \omega$ and $\tau \supseteq\{\cdot,+, \backslash\}$, the $\tau$-reduct $\mathfrak{D}_{n}$ of $\mathfrak{A}_{n}$ is not in the variety $\mathrm{V}(\tau)$ generated by $\mathrm{R}(\tau)$, since there is an equation $e_{n}$ in the language $\{\cdot,+, \backslash\}$ such that $e_{n}$ fails in $\mathfrak{D}_{n}$, but $e_{n}$ is valid in $\mathrm{R}(\tau)$, hence in $\mathrm{V}(\tau)$ (Lemma 3.1.) 
2. any nontrivial ultraproduct of $\mathfrak{A}_{n}$ is representable (is in RRA), whence the ultraproduct of $\mathfrak{D}_{n}$ is in $\mathrm{Q}^{c d}(\tau) \subseteq \mathrm{V}^{c d}(\tau)$ (Lemma 3.10.).

Hence the complements of the classes $\mathrm{Q}(\tau), \mathrm{V}(\tau), \mathrm{Q}^{c d}(\tau)$, and $\mathrm{V}^{c d}(\tau)$ are not closed under ultraproducts, and the result follows by Łoś theorem.

§3. Nonfinite axiomatizability. The rest of the paper is devoted to make the proof of Theorem 2.3. complete.

3.1. Rainbow algebras. It is, in general, very difficult to know whether a given relation algebra is representable, indeed for finite algebras this question is undecidable (Hirsch \& Hodkinson, 2001b). Nevertheless, special types of relation algebras can be defined where it is relatively easy to determine if the algebra is representable or not. The idea is to define relation algebras so that it is not too hard to work out how many rounds the second player can survive in a game to test representability (see Definition 3.2. below). A group of such constructions goes under the name of rainbow algebra, mainly because each nonidentity atom of these algebras has a color-green, yellow, white, black, or red. Rainbow algebras have been used to solve many combinatorial problems for relation algebras and their fragments (Hirsch, 1995; Hirsch \& Hodkinson, 1997, 2000, 2001a; Hodkinson, 1997; Hodkinson \& Mikulás, 2000) and there is a version for cylindric algebras (Hirsch \& Hodkinson, 2009). It is typically the case with these algebras that the first player $(\forall)$ can use green and yellow atoms to force the existence of a large set of points where each distinct pair of points from this set is forced (by the consistency rules) to be labeled by a red atom. In Hirsch \& Hodkinson (2002, Definition 16.10) these sets are called red cliques. The second player $(\exists)$ is allowed to choose, within the constraints of consistency, which red atom to use. We will see that the constraints in the finite algebras $\mathfrak{A}_{n}$ defined below restrict the moves of $\exists$ so that she eventually loses the representability game, whence $\mathfrak{A}_{n}$ is not representable. But as $n$ grows, she can survive more and more rounds in the game played on $\mathfrak{A}_{n}$, and in fact $\exists$ can win the game played on the ultraproduct $\mathfrak{A}$ of $\mathfrak{A}_{n}(n \in \omega)$, whence $\mathfrak{A}$ is representable.

Rainbow algebras are integral, but commutativity, symmetry, and density may fail. It is not hard to make rainbow algebras symmetric (hence commutative) and a symmetric relation algebra with a rule like (20) below was defined in Hodkinson \& Mikulás (2000). In the definition below we modify the construction so that the algebras are also dense, this type of construction appeared in Mikulás (2009). In these modified rainbow algebras, it is possible for player $\forall$ to make a series of moves using green and yellow atoms to force a large set of points where nearly all pairs of distinct points from the set are labeled by a red atom, but some edges may be labeled by a yellow atom. We call these sets 'red clusters', and the strategy to deal with them is new. We will explain this more carefully in the remainder of this section.

We define relation algebras $\mathfrak{A}_{n}$ for $n \in \omega$. Let $n$ be any natural number $n=\{0,1, \ldots$, $n-1\}$. We define $\mathfrak{A}_{n}$ to be the finite relation algebra (in RA) with the following atoms $\operatorname{At}\left(\mathfrak{A}_{n}\right)$ :

- identity: $1^{\prime}$

- greens: $g_{i}$ for $i \in n+1$

- yellows: $\mathrm{y}_{i}$ for $i \in n$

- blacks: $\mathrm{b}_{i}$ for $i \in n$

- whites: $\mathrm{w}_{S}$ for $S \subseteq(n+1) \times n,|S| \leq 2$

- reds: $\mathrm{r}_{i}$ for $i \in n$ 
All the atoms are self-converse. Given this, a triple $(x, y, z)$ of atoms is said to be an inconsistent (or forbidden) triangle if $x \cdot(y ; z)=y \cdot(z ; x)=z \cdot(x ; y)=0$. Using additivity, composition is determined by specifying that the set of inconsistent triangles are precisely the permutations of the following:

$$
\begin{aligned}
\left(1^{\prime}, x, y\right) & \text { unless } x=y \\
\left(\mathrm{~g}_{i}, \mathrm{~g}_{j}, \mathrm{y}_{k}\right) & \text { unless }|i-j|=1 \\
\left(\mathrm{~g}_{i}, \mathrm{~g}_{j}, \mathrm{~g}_{k}\right) & \text { unless } i=j=k \\
\left(\mathrm{~g}_{i}, \mathrm{~g}_{j}, \mathrm{w}_{S}\right) & \\
\left(\mathrm{g}_{i}, \mathrm{~g}_{j}, \mathrm{r}_{k}\right) & \text { unless } k=|i-j| \leq 5 \text { or } 5<|i-j| \equiv_{5} k \\
\left(\mathrm{y}_{i}, \mathrm{y}_{j}, \mathrm{~b}_{k}\right) & \\
\left(\mathrm{y}_{i}, \mathrm{y}_{j}, \mathrm{y}_{k}\right) & \text { unless } i=j=k \\
\left(\mathrm{y}_{i}, \mathrm{r}_{j}, \mathrm{~b}_{k}\right) & \text { unless } i=k \\
\left(\mathrm{~g}_{i}, \mathrm{y}_{j}, \mathrm{w}_{S}\right) & \text { unless }(i, j) \in S \\
\left(\mathrm{r}_{i}, \mathrm{r}_{j}, \mathrm{r}_{k}\right) & \text { unless } i=j=k \text { or } i+j=k \text { or } i+k=j \text { or } j+k=i
\end{aligned}
$$

where $\equiv 5$ denotes equivalence modulo 5 . For any three integers $i, j, k$ we define $T(i, j, k)$ to mean that either $i=j=k, i+j=k, i+k=j$, or $j+k=i$. So (20) says that $\left(\mathrm{r}_{i}, \mathrm{r}_{j}, \mathrm{r}_{k}\right)$ is forbidden unless $T(i, j, k)$. We might also use the phrase 'the indices add up' in case $T(i, j, k)$. The set of consistent triangles of atoms is denoted by $C$ :

$$
C=\left\{(x, y, z): x, y, z \in \operatorname{At}\left(\mathfrak{A}_{n}\right),(x, y, z) \text { is consistent }\right\}
$$

Note that $\mathfrak{A}_{n}$ is a dense $(x \leq x ; x)$, commutative $(x ; y=y ; x)$, integral $(x ; y=0$ implies $x=0$ or $y=0$, hence $1^{\prime}$ is an atom), and symmetric $\left(x^{\smile}=x\right)$ algebra. Also, for any natural numbers $i, j, k$, if $i \leq j \leq k$, then $|j-i|+|k-j|=|k-i|$, hence for arbitrary $i, j, k$ if $|i-j|,|j-k|,|i-k|<n$, then the triangle

$$
\left(\mathrm{r}_{|j-i|}, \mathrm{r}_{|k-j|}, \mathrm{r}_{|k-i|}\right)
$$

is consistent.

It is not difficult to check that $\mathfrak{A}_{n}$ is indeed a relation algebra, for $n \geq 5$. All the axioms but $(R 1)$ are straightforward to check. Below we sketch why $(R 1)$ holds. It suffices to show that whenever we have atoms $a, x, y$, and $z$ such that $a \leq(x ; y) ; z$, then there is an atom $b$ such that $a \leq x ; b$ and $b \leq y ; z$. If $x=a$ and $y=z$, then we can choose $b=1^{\prime}$. Otherwise we can try to use a white atom or a black atom for $b$. The only case when this might not work is when both $x$ and $a$ are green and both $y$ and $z$ are yellow (or the other way round), say $x=\mathrm{g}_{i}, a=\mathrm{g}_{j}$ and $y=\mathrm{y}_{k}, z=\mathrm{y}_{l}$. In this case, we can choose $b=\mathrm{r}_{|i-j|(\bmod 5)}$.

3.2. Nonrepresentability. Next we show that the nonrepresentability of $\mathfrak{D}_{n}$, the $\{\cdot,+, \backslash\}$-reduct of $\mathfrak{A}_{n}$, is witnessed by an equation $e_{n}$.

LEMMA 3.1. For every $2 \leq n \in \omega$, there is an equation $e_{n}$ in the language $\{\cdot,+, \backslash\}$ such that

1. $e_{n}$ fails in the $\{\cdot,+, \backslash\}$-reduct $\mathfrak{D}_{n}$ of $\mathfrak{A}_{n}$,

2. $e_{n}$ is valid over $\mathrm{Rs}$, hence over $\mathrm{R}(\cdot,+, \backslash)$.

Hence $\mathfrak{D}_{n} \notin \mathrm{V}(\cdot,+, \backslash)$.

Proof. For each atom $\alpha \in \operatorname{At}\left(\mathfrak{A}_{n}\right)$, let $v_{\alpha}$ be a variable symbol, and let $V=\left\{v_{\alpha}\right.$ : $\left.\alpha \in \operatorname{At}\left(\mathfrak{A}_{n}\right)\right\}$ denote the collection of the above variables. Note that $V$ is finite since $\mathfrak{A}_{n}$ 
is finite. For each atom $\alpha \in \operatorname{At}\left(\mathfrak{A}_{n}\right)$ let $\overline{v_{\alpha}}$ stand for $\sum\left\{v_{\beta}: \beta \in \operatorname{At}\left(\mathfrak{A}_{n}\right) \backslash\{\alpha\}\right\}$. Let $\Omega=\sum\left\{v_{\alpha} \cdot v_{\beta}: \alpha, \beta \in \operatorname{At}\left(\mathfrak{A}_{n}\right), \alpha \neq \beta\right\}$.

For each atom $\alpha \in \operatorname{At}\left(\mathfrak{A}_{n}\right)$, we define terms $\sigma_{n}(\alpha)$ by induction.

$$
\begin{aligned}
\sigma_{0}(\alpha) & =v_{\alpha} \cdot(\Omega \backslash \Omega) \\
\sigma_{m+1}(\alpha) & =\sigma_{m}(\alpha) \cdot \prod_{\beta \in \operatorname{At}\left(\mathfrak{A}_{n}\right)}\left(v_{\beta} \backslash \sum_{(\alpha, \beta, \gamma) \in C} \sigma_{m}(\gamma)\right)
\end{aligned}
$$

Terms $\tau_{m}$ are also defined by induction:

$$
\begin{aligned}
\tau_{0} & =\overline{v_{\mathrm{g}_{0}}} \\
\tau_{m+1} & =\overline{v_{\mathrm{g}_{m+1}}}+\left(\sigma_{m}\left(\mathrm{y}_{m}\right) \backslash \tau_{m}\right)
\end{aligned}
$$

We can now define the equation $e_{n}$ as

$$
\sigma_{n}\left(g_{n}\right) \leq \tau_{n}
$$

For Item 1 , let $l$ be the evaluation of the variables that maps $v_{\alpha}$ to $\alpha$, for $\alpha \in \operatorname{At}\left(\mathfrak{A}_{n}\right)$. Note that $l(\Omega)=0$ and $l\left(\overline{v_{\alpha}}\right)=-l\left(v_{\alpha}\right)=-\alpha$, for any $\alpha \in \operatorname{At}\left(\mathfrak{A}_{n}\right)$. We will show that $\imath\left(\sigma_{n}\left(\mathrm{~g}_{n}\right)\right)=\mathrm{g}_{n}$ and $\imath\left(\tau_{n}\right)=-\mathrm{g}_{n}$, whence $e_{n}$ fails in $\mathfrak{D}_{n}$.

For any $\alpha \in \operatorname{At}\left(\mathfrak{A}_{n}\right)$, we have $l\left(\sigma_{0}(\alpha)\right)=l\left(v_{\alpha} \cdot \Omega \backslash \Omega\right)=\alpha \cdot 0 \backslash 0=\alpha$, and inductively

$$
l\left(\sigma_{m+1}(\alpha)\right)=\alpha \cdot \prod_{\beta \in \operatorname{At}\left(\mathfrak{A}_{n}\right)}\left(\beta \backslash \sum_{(\alpha, \beta, \gamma) \in C} \gamma\right)=\alpha
$$

since $\alpha ; \beta=\beta ; \alpha \leq \sum_{(\alpha, \beta, \gamma) \in C} \gamma$, whence $\alpha \leq \beta \backslash \sum_{(\alpha, \beta, \gamma) \in C} \gamma$ by equivalence (7). Hence $l\left(\sigma_{n}(\alpha)\right)=\alpha$, and in particular $l\left(\sigma_{n}\left(g_{n}\right)\right)=g_{n}$.

Next we show that $l\left(\tau_{m}\right)=-\mathrm{g}_{m}$, for $m \leq n$, by induction over $m$. We have $l\left(\tau_{0}\right)=$ $l\left(\overline{v_{0}}\right)=-\mathrm{g}_{0}$. By the previous paragraph, $l\left(\sigma_{m}\left(\mathrm{y}_{m}\right)\right)=\mathrm{y}_{m}$. Then, inductively,

$$
\imath\left(\tau_{m+1}\right)=-\mathrm{g}_{m+1}+\left(\mathrm{y}_{m} \backslash-\mathrm{g}_{m}\right)=-\mathrm{g}_{m+1}
$$

since $\mathrm{g}_{m+1} \not \leq \mathrm{y}_{m} \backslash-\mathrm{g}_{m}$ by $\left(\mathrm{y}_{m}, \mathrm{~g}_{m+1}, \mathrm{~g}_{m}\right) \in C$.

For Item 2 , assume that we have a representable algebra $\mathfrak{D} \in R(\cdot,+, \backslash)$. We have to show that $e_{n}$ is valid in $\mathfrak{D}$. We will establish this by reductio ad absurdum. To this end, assume that we have a valuation $\imath: V \rightarrow D$ and a pair $\left(u_{n}, v\right)$ such that $\left(u_{n}, v\right) \in \imath\left(\sigma_{n}\left(g_{n}\right)\right)$ but $\left(u_{n}, v\right) \notin l\left(\tau_{n}\right)$. See Figure 2 for the argument below.

Since $\left(u_{n}, v\right) \notin l\left(\tau_{n}\right)$, we have $\left(u_{n}, v\right) \notin l\left(\sigma_{n-1}\left(\mathrm{y}_{n-1}\right)\right) \backslash l\left(\tau_{n-1}\right)$, so there is $u_{n-1}$ with $\left(u_{n-1}, u_{n}\right) \in \imath\left(\sigma_{n-1}\left(\mathrm{y}_{n-1}\right)\right)$ and $\left(u_{n-1}, v\right) \notin l\left(\tau_{n-1}\right)$. Continuing in the same way, there are $u_{0}, u_{1}, \ldots, u_{n}$ such that

$$
\left(u_{i}, u_{i+1}\right) \in l\left(\sigma_{i}\left(\mathrm{y}_{i}\right)\right) \text { and }\left(u_{i}, v\right) \notin l\left(\tau_{i}\right)
$$

In particular, $\left(u_{i}, u_{i+1}\right) \in \imath\left(v_{\mathrm{y}_{i}}\right)=\mathrm{y}_{i}$, since $\imath\left(v_{\mathrm{y}_{i}}\right) \supseteq \imath\left(\sigma_{i}\left(\mathrm{y}_{i}\right)\right)$, and $\left(u_{i}, v\right) \notin \imath\left(\overline{v_{g_{i}}}\right)$, since $\imath\left(\tau_{i}\right) \supseteq \imath\left(\overline{\mathrm{vg}_{i}}\right)$.

We noted that $\left(u_{i}, v\right) \notin \imath\left(\overline{v_{g_{i}}}\right)$. It follows that $\left(u_{i}, v\right) \notin l\left(v_{\alpha}\right)$ for any $\alpha \in \operatorname{At}\left(\mathfrak{A}_{n}\right)$ such that $\alpha \neq g_{i}$, whence $\left(u_{i}, v\right) \notin l(\Omega)$ by the definition of $\Omega$.

We claim that

$$
\left(u_{i}, v\right) \in \imath\left(\sigma_{i}\left(g_{i}\right)\right)
$$

for every $i \in n+1$. We know that $\left(u_{n}, v\right) \in \imath\left(\sigma_{n}\left(\mathrm{~g}_{n}\right)\right)$ by assumption. This implies $\left(u_{n}, v\right) \in \prod_{\alpha \in \operatorname{At}\left(\mathfrak{A}_{n}\right)}\left(v_{\alpha} \backslash \sum_{\left(\alpha, \mathrm{g}_{n}, \gamma\right) \in C} l\left(\sigma_{n-1}(\gamma)\right)\right)$. Since $\left(u_{n-1}, u_{n}\right) \in l\left(v_{\mathrm{y}_{n-1}}\right)$, by considering $\alpha=\mathrm{y}_{n-1}$ and the interpretation of $\backslash$ in representable algebras, we deduce that 


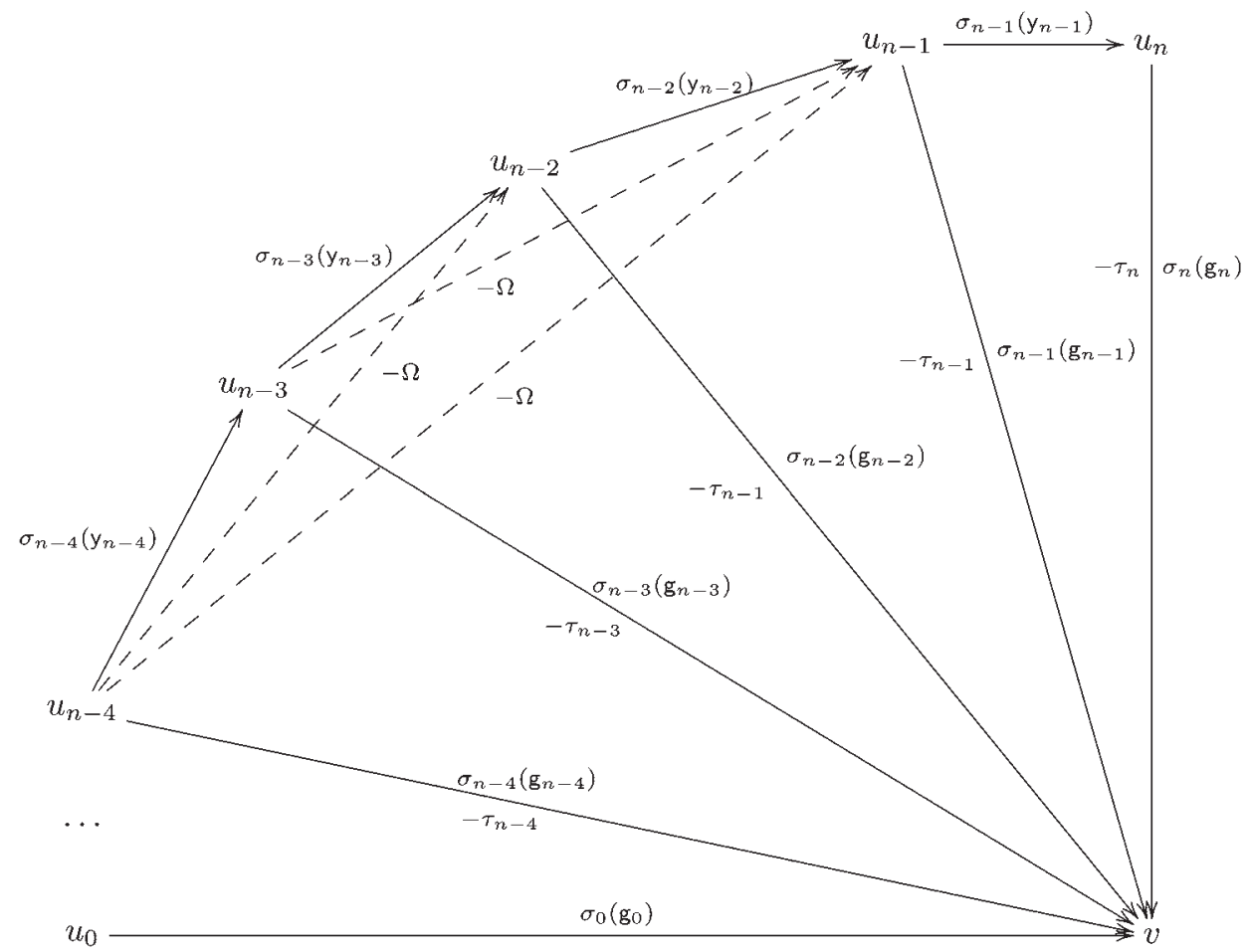

Fig. 2. The sequence of points.

$\left(u_{n-1}, v\right) \in \imath\left(\sigma_{n-1}(\gamma)\right)$, for some $\gamma \in \operatorname{At}\left(\mathfrak{A}_{n}\right)$ such that $\left(\mathrm{y}_{n-1}, \mathrm{~g}_{n}, \gamma\right) \in C$. We know that $\left(u_{n-1}, v\right) \notin l\left(\overline{v_{g_{n-1}}}\right)$, hence $\gamma=\mathrm{g}_{n-1}$ and $\left(u_{n-1}, v\right) \in \imath\left(\sigma_{n-1}\left(\mathrm{~g}_{n-1}\right)\right)$. The claim follows inductively.

Our next claim is that

$$
\left(u_{i}, u_{j}\right) \notin u(\Omega)
$$

for $i<j \leq n$. In passing we note that from this claim it follows that all the points $u_{i}$ are distinct. We have already seen that $\left(u_{i}, v\right) \notin u(\Omega)$. Suppose for contradiction that $\left(u_{i}, u_{j}\right) \in l(\Omega)$, for some $i<j \leq n$. If $j<n$, then $\left(u_{j}, u_{j+1}\right) \in l\left(\sigma_{j}\left(\mathrm{y}_{j}\right)\right) \subseteq l(\Omega \backslash \Omega)$. Since $\left(u_{i}, u_{j}\right) \in l(\Omega)$ by the indirect assumption, we get $\left(u_{i}, u_{j+1}\right) \in \Omega$. Repeating this, we deduce that $\left(u_{i}, u_{n}\right) \in l(\Omega)$. Repeating one extra time, since $\left(u_{n}, v\right) \in l(\Omega \backslash \Omega)$, we get $\left(u_{i}, v\right) \in \imath(\Omega)$, a contradiction.

Next we claim that, for each $i<j \leq n$, there is a unique atom $\alpha_{i j} \in \operatorname{At}\left(\mathfrak{A}_{n}\right)$ such that

$$
\left(u_{i}, u_{j}\right) \in \imath\left(\sigma_{i}\left(\alpha_{i j}\right)\right) \subseteq \imath\left(v_{\alpha_{i j}}\right)
$$

and for any $i<k<j \leq n$,

$$
\left(\alpha_{i k}, \alpha_{k j}, \alpha_{i j}\right) \in C
$$

First recall that $\left(u_{i}, u_{j}\right) \notin l(\Omega)$, hence such an atom $\alpha_{i j}$, if it exists at all, is unique. We have already seen that $\left(u_{i}, u_{i+1}\right) \in \imath\left(\sigma_{i}\left(\mathrm{y}_{i}\right)\right) \subseteq \imath\left(v_{\mathrm{y}_{i}}\right)$, so

$$
\alpha_{i, i+1}=\mathrm{y}_{i}
$$

for $i<n$. Now assume that $|j-i| \geq 2$ and let $i<k<j$. Then, inductively, $\left(u_{k}, u_{j}\right) \in$ $l\left(\sigma_{k}\left(\alpha_{k j}\right)\right) \subseteq l\left(\prod_{\alpha \in \operatorname{At}\left(\mathfrak{A}_{n}\right)}\left(v_{\alpha} \backslash \sum_{\left(\alpha, \alpha_{k j}, \gamma\right) \in C} \sigma_{k-1}(\gamma)\right)\right) \subseteq l\left(v_{\alpha_{i k}} \backslash \sum_{\left(\alpha_{i k}, a_{k j}, \gamma\right) \in C} \sigma_{k-1}(\gamma)\right)$. 
By the inductive hypothesis, we have $\left(u_{i}, u_{k}\right) \in l\left(v_{\alpha_{i k}}\right)$. Then by the definition of $\backslash$ in representable algebras, $\left(u_{i}, u_{j}\right) \in l\left(\sigma_{k-1}(\gamma)\right)$ for some $\gamma=\alpha_{i j}$ such that the triple $\left(\alpha_{i k}, \alpha_{k j}, \alpha_{i j}\right) \in C$. Since $i \leq k-1$, we get $\left(u_{i}, u_{j}\right) \in l\left(\sigma_{k-1}(\gamma)\right) \subseteq l\left(\sigma_{i}(\gamma)\right) \subseteq$ $l\left(v_{\gamma}\right)=\gamma$.

We claim that

$$
\left(\alpha_{i j}, g_{j}, g_{i}\right) \in C
$$

for $i<j \leq n$ as well. Since $\left(u_{j}, v\right) \in \imath\left(\sigma_{j}\left(\mathrm{~g}_{j}\right)\right)$, we have $\left(u_{j}, v\right) \in \imath\left(v_{\mathrm{g}_{j}} \cdot\left(v_{\alpha_{i j}} \backslash\right.\right.$ $\left.\left.\sum_{\left(\alpha_{i j}, \mathrm{~g}_{j}, \gamma\right) \in C} v_{\gamma}\right)\right)$. Recall that $\left(u_{i}, u_{j}\right) \in l\left(v_{\alpha_{i j}}\right)$. Hence $\left(u_{i}, v\right) \in l\left(v_{\gamma}\right) \in \operatorname{At}\left(\mathfrak{A}_{n}\right)$ such that $\left(\alpha_{i j}, \mathrm{~g}_{j}, \gamma\right) \in C$. But we have seen that $\left(u_{i}, v\right) \notin l\left(\overline{v_{\mathrm{g}_{i}}}\right)$, hence $\gamma=\mathrm{g}_{i}$ and $\left(\alpha_{i j}, \mathrm{~g}_{j}, \mathrm{~g}_{i}\right) \in C$ as desired.

Next we claim that for $i+2 \leq j \leq n$,

$$
\alpha_{i j}=\mathrm{r}_{j-i}
$$

The case $i=0, j=n$ will then provide a contradiction, since there is no atom $r_{n}$. See Figure 3 (where we use the notation $l\left(v_{\alpha}\right)=\alpha$ for any variable $v_{\alpha}$ with $\alpha \in \operatorname{At}\left(\mathfrak{A}_{n}\right)$ ) for the argument below. We will use that certain triples of elements must satisfy the consistency conditions on atomic triangles on $\mathfrak{A}_{n}$, see the claims (22) and (24) above.

We prove the claim by induction over $j-i$, the base case is $j-i=2$. We know that $\alpha_{i, i+1}=\mathrm{y}_{i}$ and $\alpha_{i+1, i+2}=\mathrm{y}_{i+1}$ by (23), so $\left(\mathrm{y}_{i}, \mathrm{y}_{i+1}, \alpha_{i, i+2}\right) \in C$ by (22). Thus $\alpha_{i, i+2}$ cannot be the identity, yellow or black by (11), (17), and (16). We also know that $\left(\alpha_{i, i+2}, g_{i}, g_{i+2}\right) \in C$ by (24). So $\alpha_{i, i+2}$ cannot be green or white by (13) and (14). Hence it must be red. By (15), the only possibility is $\alpha_{i, i+2}=r_{2}$, proving the base case.

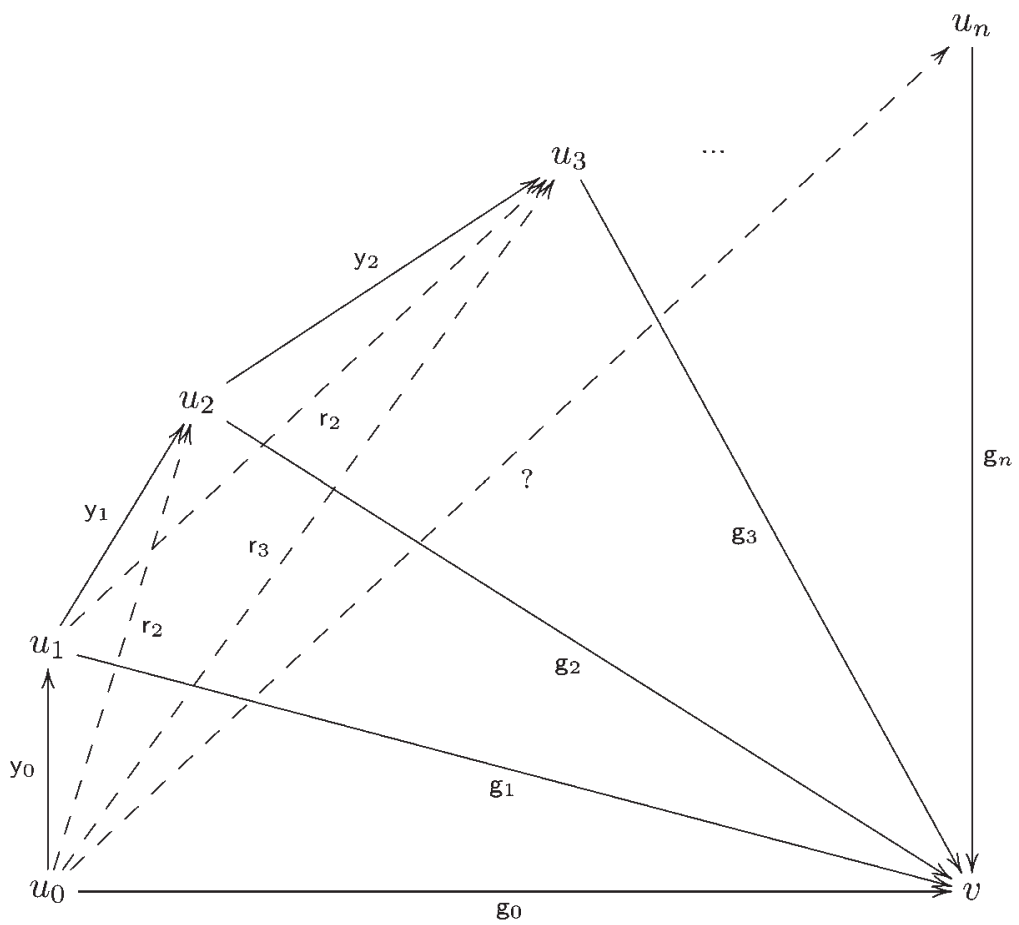

Fig. 3. Red arrows. 
Next we suppose $i<j \leq n$ and $j=i+3 \leq n$, and we consider the atom $\alpha_{i, i+3}$. We already have $\alpha_{i, i+2}=\alpha_{i+1, i+3}=\mathrm{r}_{2}$ and $\alpha_{i, i+1}=\mathrm{y}_{i}$ and $\alpha_{i+2, i+3}=\mathrm{y}_{i+2}$. So $\alpha_{i, i+3}$ must satisfy

$$
\left(\mathrm{y}_{i}, \mathrm{r}_{2}, \alpha_{i, i+3}\right),\left(\mathrm{r}_{2}, \mathrm{y}_{i+2}, \alpha_{i, i+3}\right),\left(\alpha_{i, i+3}, \mathrm{~g}_{i}, \mathrm{~g}_{i+3}\right) \in C
$$

and hence $\alpha_{i, i+3}=\mathrm{r}_{3}$, by (13), (12), (14), and (15). Similarly, $\alpha_{i j}=\mathrm{r}_{j-i}$ whenever $i<j \leq n$ and $j-i \leq 5$.

Now let $i<j \leq n$ and $5<j-i$. Assume, as an induction hypothesis, that $\alpha_{i^{\prime}, j^{\prime}}=\mathrm{r}_{j^{\prime}-i^{\prime}}$ whenever $i^{\prime}<j^{\prime} \leq n$ and $j^{\prime}-i^{\prime}<j-i$. As before, $\alpha_{i j}$ cannot be the identity, black, yellow, green, or white, so $\alpha_{i j}=\mathrm{r}_{m}$, for some $m<n$. By (24), $\left(\mathrm{r}_{m}, \mathrm{~g}_{i}, \mathrm{~g}_{j}\right) \in C$, and by (15) it follows that $m \equiv_{5} j-i$. Also, by (22), $\left(\mathrm{r}_{2}, \mathrm{r}_{j-i-2}, \mathrm{r}_{m}\right) \in C$, and hence by (20) the indices must add up: $T(2, j-i-2, m)$. That is, either (i) $m=2=j-i-2$, or (ii) $m=(j-i-2)+2(=j-i)$, or (iii) $j-i-2=2+m$ (so $j-i-4=m$ ), or (iv) $2=m+(j-i-2)$. The first alternative is impossible, since $j-i-2 \geq 3>2$. The fourth alternative is impossible, since $m \geq 0$ and $j-i-2>5-2=3>2$. The third alternative is also impossible, since $m \equiv_{5} j-i$ but $j-i-4 \not \equiv_{5} j-i$. Hence it is the second alternative that must hold, and thus $\alpha_{i j}=\mathrm{r}_{j-i}$. This completes the induction step.

In particular, we would have $\left(u_{0}, u_{n}\right) \in \imath\left(v_{\rho}\right)$ for a red atom $\rho$ with index $n$, but $r_{n}$ does not exist. We have arrived at a contradiction, hence $e_{n}$ holds in $\mathfrak{D}$.

To complete the proof of Theorem 2.3. it remains to show that an ultraproduct of $\mathfrak{A}_{n}$ is representable. To this end we recall from Hirsch \& Hodkinson (1997) a (variant of the) game connected to representability.

\subsection{The game.}

DEFINITION 3.2. Let $\mathfrak{A}$ be a relation-type algebra.

1. A network is a complete, directed, finite graph with edges labeled by atoms of $\mathfrak{A}$ : that is, $N=\left(N_{1}, N_{2}\right)$ for some set $N_{1}$ (the set of nodes) and some map $N_{2}$ : $N_{1} \times N_{1} \rightarrow \operatorname{At}(\mathfrak{A})$ that satisfies, for every $x, y, z \in N_{1}$,

(a) $N_{2}(x, y) \leq 1^{\prime}$ iff $x=y$,

(b) $N_{2}(x, y) ; N_{2}(y, z) \geq N_{2}(x, z)$.

Given two networks $N=\left(N_{1}, N_{2}\right)$ and $N^{\prime}=\left(N_{1}^{\prime}, N_{2}^{\prime}\right)$, we write $N \subseteq N^{\prime}$ if $N_{1} \subseteq N_{1}^{\prime}$ and for all $x, y \in N_{1}, N_{2}^{\prime}(x, y)=N_{2}(x, y)$.

If no confusion is likely, we will henceforth omit the subscripts and let $N$ denote the network, the set of nodes and the labeling function, distinguishing cases by context. Sometimes, when confusion is possible, we may write nodes $(N)$ for the set of nodes $N_{1}$ of the network.

2. Let $t \leq \omega$. We define a game $\mathcal{G}_{t}(\mathfrak{A})$ between two players, $\forall$ (male), and $\exists$ (female). During a match of the game, they build a finite chain $N_{0} \subseteq N_{1} \subseteq \cdots \subseteq N_{t}$ of networks in the following way. In the initial round $\forall$ picks any atom $\alpha$ and $\exists$ plays a network $N_{0}$ containing nodes $m_{0}, n_{0}$ such that $N\left(m_{0}, n_{0}\right)=\alpha$ (if $\alpha \leq 1^{\prime}$, then $m_{0}=n_{0}$, else $\left.m_{0} \neq n_{0}\right)$. If $m_{0} \neq n_{0}$, the edges $\left(m_{0}, n_{0}\right)$ and $\left(n_{0}, m_{0}\right)$ belong to $\forall$. In a subsequent round $i(i<t)$, after $N_{i-1}$ has been played,

- $\forall$ chooses an edge $(m, n)$ from $N_{i-1}$ and atoms $x, y \in \operatorname{At}(\mathfrak{A})$ such that $x ; y \geq N_{i-1}(m, n)$

- $\exists$ responds with a network $N_{i} \supseteq N_{i-1}$ containing a node $l$ such that $N_{i}(m$, $l)=x$ and $N_{i}(l, n)=y$. 
For irreflexive edges of $N_{i}$ where both nodes belong to $N_{i-1}$, the owner of the edge in $N_{i}$ is the same as it was in $N_{i-1}$. The edges $(m, l),(l, n)$ and their converses belong to $\forall$. All other irreflexive edges in $N_{i}$ belong to $\exists$. $\exists$ wins a match of the game $\mathcal{G}_{t}(\mathfrak{A})$ if she can respond to every move of $\forall$ by a network $N_{i}$, for every natural number $i \leq t$. We say that $\exists$ has a winning strategy if she can win all matches.

The following proposition (Hirsch \& Hodkinson, 1997, Proposition 15) provides us with a sufficient condition for representability of atomic relation algebras.

PROPOSITION 3.3. Let $\mathfrak{A}$ be an atomic relation algebra. Then $\exists$ has a winning strategy in $\mathcal{G}_{t}(\mathfrak{A})$ for all $t \in \omega$ iff $\mathfrak{A}$ is elementarily equivalent to a completely representable relation algebra. ${ }^{3}$ Hence, because RRA is elementary, if $\exists$ has a winning strategy in $\mathcal{G}_{t}(\mathfrak{A})$ for all $t \in \omega$, then $\mathfrak{A}$ is representable.

DEFINITION 3.4. Let $N$ be a network over $\mathfrak{A}_{n}$ and let $x \in N$. Define

$$
\begin{aligned}
& \mathrm{G}_{N}(x)=\{y \in N: N(x, y) \text { is green }\} \\
& \mathbf{Y}_{N}(x)=\{y \in N: N(x, y) \text { is yellow }\}
\end{aligned}
$$

If $\mathrm{G}_{N}(x)$ is nonempty, define an equivalence relation $\sim_{x}$ over $\mathrm{G}_{N}(x)$ by letting $y \sim_{x} z$ iff there is a yellow path $\left(\rho_{0}, \ldots, \rho_{k}\right)$ contained in $\mathrm{G}_{N}(x)$, where $y=\rho_{0}, z=\rho_{k}$, and $N\left(\rho_{i}, \rho_{i+1}\right)$ is yellow for every $i<k$.

Certain subsets of $X \subseteq \operatorname{nodes}(N)$ are called red clusters. There are two types (see Figure 4). The set $X$ is a red cluster if $|X| \geq 2$ and either

Type 1: there are distinct nodes $x, y \in N$ such that $N(x, y)$ is not green or white (the case where $N(x, y)$ is green is covered by the other type of red cluster, the case where $N(x, y)$ is white is not a red cluster and is handled separately) and

$$
X=\mathrm{G}_{N}(x) \cap \mathrm{Y}_{N}(y)
$$

Type 2: there is $x \in N$ and $X$ is a $\sim_{x}$-equivalence class of $\mathrm{G}_{N}(x)$.

If $X$ is a red cluster and $X=\mathrm{G}_{N}(x) \cap \mathrm{Y}_{N}(y)$ or $X$ is a $\sim_{x}$-equivalence class of $G_{N}(x)$ ( some $x, y$ ), then $x$ is called a green root of the red cluster $X$. If $X=\mathrm{G}_{N}(x) \cap \mathrm{Y}_{N}(y)$ is a red cluster of the second type, the node $y$ is called a yellow root of $X$. By definition, every red cluster has a green root, but we do not assume that it has a unique green root.

Let $s \in \omega$ and let $x \in N$. If $X \subseteq \mathrm{G}_{N}(x)$, then an $s$-good map $f$ over $x$ is a map $f: X \rightarrow(n+1)$ such that for $y, z \in X$ if $N(x, y)=g_{i}$ and $N(x, z)=g_{j}($ some $i, j \leq n)$, then (i) $i \leq j \Longleftrightarrow f(y) \leq f(z)$, (ii) $f(y)-f(z) \equiv_{5} j-i$, (iii) if $|i-j|<6 \cdot 2^{s}$, then $f(y)-f(z)=i-j$, and (iv) if $|i-j| \geq 6 \cdot 2^{s}$, then $|f(y)-f(z)| \geq 6 \cdot 2^{s}$.

Observe by the definition of red cluster above, that the red clusters of the network $N$ are completely determined by the sets of green, yellow, and white edges of $N$, in other words if $N, N^{\prime}$ are two networks with the same set of nodes and for all $u, v \in \operatorname{nodes}(N), N(u, v)$ is green (yellow, white) if and only if $N^{\prime}(u, v)$ is green (respectively yellow, white), then for any $X \subseteq \operatorname{nodes}(N), X$ is a red cluster of $N$ iff $X$ is a red cluster of $N^{\prime}$, and $x$ is a green

3 A complete representation of a relation algebra $\mathfrak{B}$ is an isomorphism from $\mathfrak{B}$ to a representable relation algebra that preserves arbitrary meets and joins whenever they exist in $\mathfrak{B}$. The algebras considered here are finite, and for finite algebras representability is the same as complete representability. 


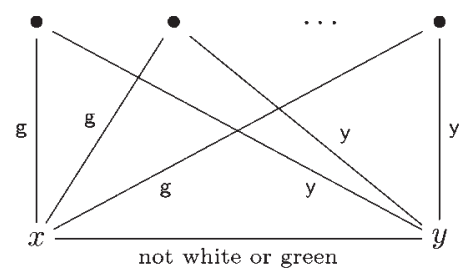

(a)

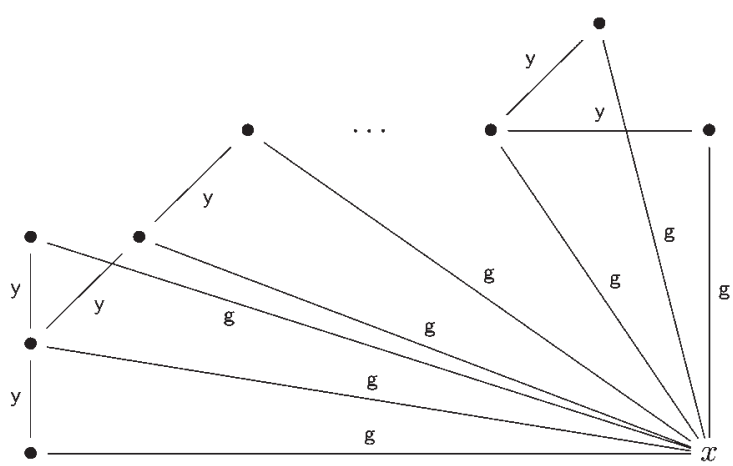

(b)

Fig. 4. Type 1 and 2 red clusters.

(or yellow) root of the red cluster $X$ in $N$ iff it is a green (or yellow) root of the same red cluster in $N^{\prime}$.

The following proposition can be shown by a standard binary chop argument (cf. Hodkinson \& Mikulás, 2000).

Proposition 3.5. Let $x \in N, X \subseteq \mathrm{G}_{N}(x), f: X \rightarrow(n+1)$ be an s-good map over $x$ and $l \in \mathrm{G}_{N}(x) \backslash X$. Then there is an $(s-1)$-good map $f^{+}: X \cup\{l\} \rightarrow(n+1)$ over $x$ extending $f$. Furthermore, if $n \geq 6 \cdot 2^{t}$ and $|X|=2$, then there is a $t$-good map $f: X \rightarrow(n+1)$.

3.4. The ultraproduct. We will now show that an ultraproduct of the $\mathfrak{A}_{n}(n \in \omega)$ is representable. First we describe a winning strategy for $\exists$ in the game $\mathcal{G}_{t}\left(\mathfrak{A}_{n}\right)$ with $6 \cdot 2^{t} \leq n$.

Suppose after $s<t$ rounds of $\mathcal{G}_{t}\left(\mathfrak{A}_{n}\right)$ the network $N_{s}$ has just been played. In the next round $\forall$ picks nodes $m, n \in N_{s}$ and atoms $\alpha, \beta$ such that $\alpha ; \beta \geq N(m, n)$. We assume the following.

Inductive hypothesis: For any red cluster $X$ and any green root $p$ of $X$, there is an associated $(t-s)$-good function $f=f_{N, X, p}: X \rightarrow(n+1)$ over $p$, and for all distinct $y, z \in X$ either $N(y, z)$ is yellow or $N(y, z)=\mathbf{r}_{|f(y)-f(z)|}$.

$\exists$ 's strategy is to follow the first applicable of the instructions below.

1. If there is a node $l \in N_{s}$ such that $N_{s}(m, l)=\alpha$ and $N_{s}(l, n)=\beta$, then she does not extend $N_{s}$.

Suppose there is no such node in $N_{s}$. In each of the following cases, $\exists$ will extend $N_{s}$ to $N_{s+1}$ containing exactly one extra node $l$ such that $N_{s+1}(m, l)=\alpha$ and $N_{s+1}(l, n)=\beta$. To define $N_{s+1}$ she has to define each label $N_{s+1}(l, p)$ for $p \in N_{s} \backslash\{m, n\}$ (she lets $N_{s+1}(p, l)=N_{s+1}(l, p)$, since the algebra is symmetric). She only ever uses white, black, or red for her labels.

2. For each $p \in N_{s}$, if either $N_{s}(p, m)$ or $x$ is not green and either $N_{s}(p, n)$ or $y$ is not green, then $\exists$ lets $N_{s+1}(p, l)=\mathrm{w}_{S}$ where

$$
S=\left\{(i, j):\{N(p, m), \alpha\}=\left\{\mathrm{g}_{i}, \mathrm{y}_{j}\right\} \text { or }\{N(p, n), \beta\}=\left\{\mathrm{g}_{i}, \mathrm{y}_{j}\right\}\right\}
$$

Observe that $|S| \leq 2$. 
In the remaining cases, $\exists$ only uses black or red atoms to label edges (see Parts 3, 4, and 5 of $\exists$ 's strategy below). Hence the red clusters of $N_{s+1}$ are already determined, as we remarked. It is therefore safe to refer to a red cluster of $N_{s+1}$, although we have not yet completed the definition of $N_{s+1}$. The following proposition is a consequence of the first two parts of $\exists$ 's strategy.

PROPOSITION 3.6. Let $N$ be a network played in a match of $G_{t}\left(\mathfrak{A}_{n}\right)$ in which $\exists$ never uses green or yellow to label edges and she always uses Parts 1 and 2 of her strategy whenever they apply (other edges may be labeled by arbitrary black or red atoms). Let $X \subseteq \operatorname{nodes}(N)$ be a set of at least four nodes. In the match so far, let $x \in X$ be the node which was last to be added (we may refer to $x$ as the most recently added node of $X$ ). Then $x$ is incident with at most two edges in $X$ labeled by $\forall$. Suppose there are $u, v \in X$ such that $(u, x),(v, x)$ were labeled by $\forall$. For all $y \in X \backslash\{x, u, v\}$, (i) it is not the case that $N(x, u)=N(u, y)$ and $N(x, v)=N(v, y)$ and (ii) either $N(y, u)$ and $N(u, x)$ are both green or $N(y, v)$ and $N(v, x)$ are both green or $N(x, y)=\mathrm{w}_{S}$ where

$$
S=\left\{(i, j):\left\{\mathbf{g}_{i}, \mathbf{y}_{j}\right\}=\{N(x, u), N(u, y)\} \text { or }\left\{\mathbf{g}_{i}, \mathbf{y}_{j}\right\}=\{N(x, v), N(v, y)\}\right\}
$$

LEMMA 3.7. Let $N$ occur in a match of $\mathcal{G}_{t}\left(\mathfrak{A}_{n}\right)$ in which $\exists$ never chooses green or yellow to label edges, and she always uses Parts 1 and 2 of her strategy whenever they apply. If $X$ and $Y$ are distinct red clusters of $N$, and $u, v$ are distinct nodes in $X \cap Y$, then $N(u, v)$ is yellow.

Proof. Both red clusters $X$ and $Y$ can be of Type 1 or Type 2. First we consider two red clusters which are both of the first type, $X=\mathrm{G}_{N}(x) \cap \mathrm{Y}_{N}(y)$ and $Y=\mathrm{G}_{N}\left(x^{\prime}\right) \cap \mathrm{Y}_{N}\left(y^{\prime}\right)$, where $x, y, x^{\prime}, y^{\prime} \in N$. Since $X$ and $Y$ are clusters of the first type, we know that $N(x, y)$ and $N\left(x^{\prime}, y^{\prime}\right)$ are not white or green. In this case there cannot be two distinct nodes in the intersection of the two clusters because of the following. Suppose for contradiction that $u, v \in\left(\mathrm{G}_{N}(x) \cap \mathrm{Y}_{N}(y)\right) \cap\left(\mathrm{G}_{N}\left(x^{\prime}\right) \cap \mathrm{Y}_{N}\left(y^{\prime}\right)\right)$ where $u \neq v$. Consider the set of nodes $Z=\left\{x, y, x^{\prime}, y^{\prime}, u, v\right\}$. If $x$ is the most recently added node from this set then, by Proposition 3.6. and since $(x, u),(x, v)$ belong to $\forall, \exists$ chose $N(x, y)$ and she chose a white atom $N(x, y)=\mathrm{w}_{S}$, for some $S$, contrary to our assumptions. Similarly, $y, x^{\prime}, y^{\prime}$ cannot be the most recently added node, hence it must be $u$ or $v$. Now for all $w \in\left\{x, y, x^{\prime}, y^{\prime}\right\}, N(u, w)$ and $N(v, w)$ are green or yellow, hence $(u, w)$ and $(v, w)$ belong to $\forall$. Since either $u$ or $v$ is incident with at most two $\forall$-edges in $Z$, we must have $\left|\left\{x, y, x^{\prime}, y^{\prime}\right\}\right| \leq 2$. Hence $\{x, y\}=\left\{x^{\prime}, y^{\prime}\right\}$ as $x \neq y$ and $x^{\prime} \neq y^{\prime}$. Note that $x=y^{\prime}$ is impossible, since $N(x, u)$ is green and $N\left(y^{\prime}, u\right)$ is yellow. Similarly $y=x^{\prime}$ is impossible, hence $x=x^{\prime}$ and $y=y^{\prime}$. This contradicts our assumption that $\mathrm{G}_{N}(x) \cap \mathrm{Y}_{N}(x), \mathrm{G}_{N}\left(x^{\prime}\right) \cap$ $\mathrm{Y}_{N}\left(y^{\prime}\right)$ were distinct red clusters.

Now consider a red cluster $\mathrm{G}_{N}(x) \cap \mathrm{Y}_{N}(y)$ of the first type and a red cluster $Y$ of the second type, say $Y$ is a $\sim_{x^{\prime}}$-equivalence class of $\mathrm{G}_{N}\left(x^{\prime}\right)$, for some $x^{\prime} \in N$. For contradiction suppose $u \neq v, u, v \in\left(\mathrm{G}_{N}(x) \cap \mathrm{Y}_{N}(y)\right) \cap Y$ and $N(u, v)$ is not yellow. Let $\left(\rho_{0}, \ldots, \rho_{k}\right)$ be a yellow path from $u$ to $v$ in $\mathrm{G}_{N}\left(x^{\prime}\right)$. By our assumption that $N(u, v)$ is not yellow we know that $k \geq 2$. Consider the set $Z=\left\{x, y, x^{\prime}, \rho_{i}: i \leq k\right\}$. As before, the most recently added node from this set cannot be $x, y, x^{\prime}, \rho_{1}, \ldots, \rho_{k-1}$, either because the node is incident with three $\forall$-edges in $Z$ or, in the cases of $x$ and $y$, because it would imply, by Proposition 3.6., that $N(x, y)$ was white, contrary to assumption. Hence the most recently added node is either $u$ or $v$. But $u$ is incident with the $\forall$-edges $(u, x),(u, y),\left(u, x^{\prime}\right)$, and $\left(u, \rho_{1}\right)$, while $v$ is incident with $(v, x),(v, y),\left(v, x^{\prime}\right)$, and $\left(v, \rho_{k-1}\right)$. Since either $u$ or $v$ is incident with at most two $\forall$-edges in $Z$, either $\left\{x^{\prime}, \rho_{1}\right\} \subseteq\{x, y\}$ or $\left\{x^{\prime}, \rho_{k-1}\right\} \subseteq\{x, y\}$. 
Now, $x^{\prime}=y$ is impossible as $N(y, u)$ is yellow but $N\left(x^{\prime}, u\right)$ is green, hence $x^{\prime}=x$. Since $\rho_{i}=x$ is impossible $\left(N\left(x^{\prime}, \rho_{i}\right)\right.$ is not the identity), either $\rho_{1}=y$ or $\rho_{k-1}=y$. Either alternative, $\rho_{1}=y$ or $\rho_{k-1}=y$, is impossible as $N\left(x^{\prime}, \rho_{i}\right)$ is green but $N(x, y)$ is not green.

Finally, consider two red clusters $X$ and $Y$ of the second type, say $X$ is a $\sim_{x}$-equivalence class over $\mathrm{G}_{N}(x)$ and $Y$ is a $\sim_{y}$-equivalence class over $\mathrm{G}_{N}(y)$, for some $x, y \in N$. Let $u, v$ be distinct nodes in $X \cap Y$. Let $\left(\rho_{0}, \ldots, \rho_{k}\right)$ be a yellow path from $u$ to $v$ in $\mathrm{G}_{N}(x)$ and let $\left(\rho_{0}^{\prime}, \ldots, \rho_{m}^{\prime}\right)$ be a yellow path from $u$ to $v$ in $\mathrm{G}_{N}(y)$. Let $Z=\left\{x, y, \rho_{i}, \rho_{j}^{\prime}: i \leq\right.$ $k, j \leq m\}$. Suppose, for contradiction, that $N(u, v)$ is not yellow. Then each of the two yellow paths from $u$ to $v$ must have length at least two. But then $x, y$ and each of the internal nodes of the two yellow paths are incident with at least three $\forall$-edges in $Z$. That leaves the two external nodes of the paths, namely $u$ and $v$, as the only candidates for most recently added edge. But $u$ is incident with the $\forall$-edges $(x, u),(y, u),\left(u, \rho_{1}\right)$, and $\left(u, \rho_{1}^{\prime}\right)$ in $Z$, while $v$ is incident with the $\forall$-edges $(x, v),(y, v),\left(\rho_{k-1}, v\right)$, and $\left(\rho_{m-1}^{\prime}, v\right)$. By Proposition 3.6., either $u$ or $v$ is incident with at most two $\forall$-edges in $Z$. Hence $x=y$ and either $\rho_{1}=\rho_{1}^{\prime}$ or $\rho_{k-1}=\rho_{m-1}^{\prime}$. But then $X, Y$ are $\sim_{x}$-equivalence classes of $\mathrm{G}_{N}(x)$ with a nonempty intersection, whence identical, contradicting our assumption that $X, Y$ are distinct.

Recall that the red clusters of $N_{s+1}$ are already determined.

LEMMA 3.8. If $X$ is a red cluster of $N_{s+1}$ and $l \in N_{s+1} \backslash N_{s}$, then either

- $|X|=2$ or

- $X \backslash\{l\}$ is a red cluster of $N$.

Proof. Let $X$ be a red cluster of $N_{s+1}$ and suppose $|X|>2$. The new node $l$ cannot be a green or yellow root of $X$ since it is incident with at most two green edges and at most two yellow edges (if $l$ is a green (yellow) root of $X$, then each edge $(l, x)$ for $x \in X$ is green (yellow)). Let $x \in N_{s}$ be a green root of $X$. Since $N_{s+1}$ is an extension of $N$, the set $X \backslash\{l\}$ is a red cluster of $N$ with green root $x$.

Hence, by Proposition 3.5., we can associate a $(t-(s+1))$-good map with each red cluster of $N_{s+1}$ in such a way that if $X \backslash\{l\}$ is a red cluster of $N_{s}$ then the $(t-(s+1))$-good map associated with $X$ is an extension of the $(t-s)$-good map associated with $X \backslash\{l\}$ in $N$.

We continue with $\exists$ 's strategy. From now on, we will assume that $\alpha$ and $N_{s}(m, p)$ are both green. The case where $\beta$ and $N_{s}(n, p)$ are both green is entirely symmetric.

3. Suppose that $N_{s}(p, m)$ and $\alpha$ are green. Suppose $p$ and $l$ do not belong to the same red cluster of $N_{s+1}$. Suppose also it is not the case that $\beta$ and $N_{s}(n, p)$ are both yellow. Then $\exists$ lets $N_{s+1}(l, p)=\mathrm{b}_{j}$ for some $j<n$ determined as follows (see Figure 5).

(a) If $\beta=\mathrm{y}_{j}$ and $N_{s}(n, p)$ are red, then $\exists$ lets $N_{s+1}(p, l)=\mathrm{b}_{j}$.

(b) If $\beta$ is red and $N_{s}(n, p)=\mathrm{y}_{j}$, then $\exists$ lets $N_{s+1}(p, l)=\mathrm{b}_{j}$.

(c) If $\beta$ is yellow and there is $q \in N_{s}$ such that $N_{s}(m, q)$ is green, $N_{s}(q, n)$ is yellow and $N_{s}(p, q)=\mathrm{y}_{j}$, then $\exists$ lets $N_{s+1}(p, l)=\mathrm{b}_{j}$.

(d) Otherwise (none of the previous subcases apply) $\exists$ lets $N_{s+1}(p, l)=\mathrm{b}_{0}$.

We will check that this part of the strategy is well defined later. In the remaining cases, $\alpha$ and $N_{s}(m, p)$ are both green and either $\beta$ and $N_{S}(n, p)$ are both yellow, or $l$ and $p$ 


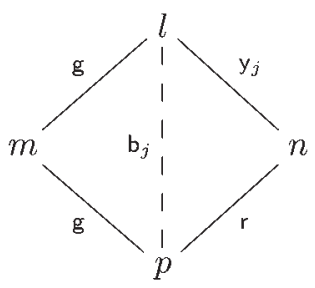

(a)

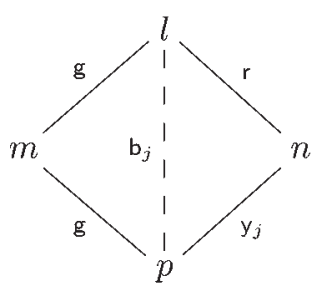

(b)

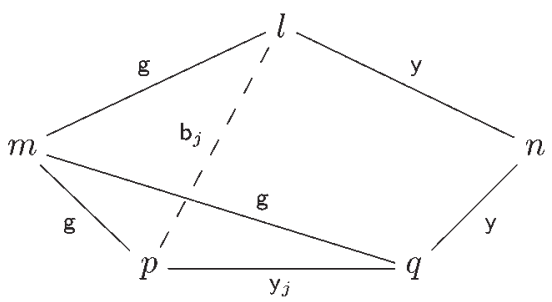

(c)

Fig. 5. Choosing a black atom to label $(l, p)$.

belong to the same red cluster of $N_{s+1}$ (both conditions might apply). In these cases $\exists$ lets $N_{s+1}(l, p)$ be a red atom.

4. If $\alpha=\mathrm{g}_{i}$ and $N_{s}(m, p)=\mathrm{g}_{j}$ are both green, $\beta$ and $N_{s}(n, p)$ are both yellow and $N_{S}(m, n)=\mathrm{w}_{S}$ (some $\left.S\right)$, then $\exists$ lets $N_{s+1}(l, p)=\mathrm{r}_{|i-j|(\bmod 5)}$.

5. Suppose that $l$ and $p$ belong to the same red cluster $X$ of $N_{s+1}$. The edge $N_{s+1}(l, p)$ will be red (not yellow) so, by Lemma 3.7., this red cluster is unique. Let $f^{+}$be the $(t-(s+1))$-good map associated with $X . \exists$ lets $N_{s+1}(l, p)=\mathrm{r}_{\left|f^{+}(l)-f^{+}(p)\right|}$.

It remains to prove that this strategy is well defined and that $N_{s+1}$ is in fact a network.

We must show that $\exists$ 's strategy is well defined in Part 3, where she chooses black atoms. We claim that when labeling the edge $(l, p)$ (some $p \in N_{s}$ ), it is impossible for two different parts from 3(a)-3(c) to apply, and if there are nodes $q, q^{\prime} \in N_{s}$ such that 3(c) applies using $q$ and also using $q^{\prime}$, then $q=q^{\prime}$. To prove this claim it is clear first of all that if 3(b) applies (so $\alpha$ and $\beta$ are green and red, respectively), then none of the other parts can apply (for Parts 3(a) and 3(c), $\alpha$ and $\beta$ are green and yellow, respectively).

Suppose, for contradiction, that 3(a) and 3(c) both apply, as in the first part of Figure 6. By 3(a), $N_{s}(m, p)$ is green, $N_{s}(n, p)$ is red, and by 3(c) there is $q \in N_{s}$ such that $N_{s}(p, q)$ is yellow, $N_{s}(n, q)=\mathrm{y}_{j}$ (some $\left.j<n\right)$ and $N_{s}(m, q)=\mathrm{g}_{i}$ (some $\left.i \leq n\right)$. We also know by

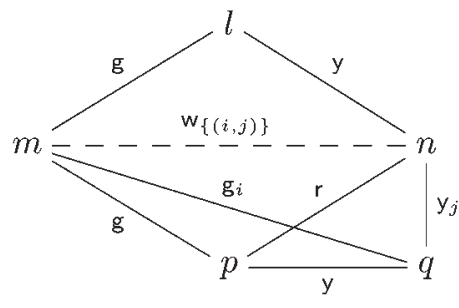

(a) and (c)

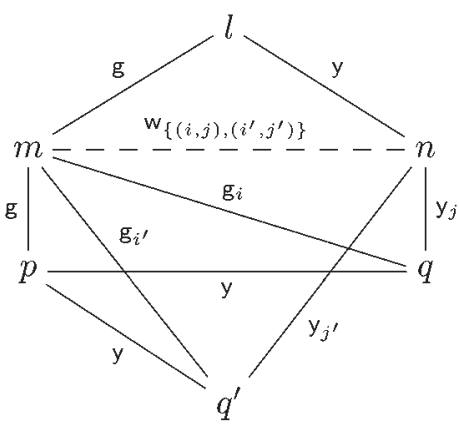

(c) and (c)

Fig. 6. If Part 3 applied in two ways. 
the conditions for Part 3 of $\exists$ 's strategy, that $l$ and $p$ do not belong to the same red cluster of $N_{s+1}$. We claim that under these conditions

$$
N_{s}(m, n)=\mathrm{W}_{\{(i, j)\}}
$$

To prove this claim, consider the nodes $\{m, n, p, q\}$. It is easily seen, by our assumptions, that the four nodes are distinct. One of these four nodes was the most recently added node from the set. By Proposition 3.6., this node is incident with at most two edges belonging to $\forall$ within that set. This most recently added node cannot be $q$, since it is incident with green/yellow edges $(q, n),(q, m)$, and $(q, p)$. If the most recent node is $p$, then by Proposition 3.6. either $N_{s}(m, n)$ is green or $N_{s}(p, n)$ is white. The latter contradicts our assumption that $N_{s}(p, n)$ is red. If $N_{s}(m, n)$ is green, then $l$ and $p$ belong to the same red cluster of $N_{s+1}$, since there is a yellow path from $l$ to $p$ in $\mathrm{G}_{N_{s+1}}(m)$, namely $(l, n, q, p)$ - again this is contrary to our assumptions. Hence the most recently added node from $\{m, n, p, q\}$ must be $m$ or $n$. If it is $m$, then by Proposition 3.6., $\exists$ would have let $N_{s}(m, n)=\mathrm{W}_{\{(i, j)\}}$. If $n$ is the most recently added node from $\{m, n, p, q\}$, then we have to consider two possibilities. It could be that $N_{s}(p, n)$ was labeled by $\forall$, but then $n$ is incident with two $\forall$-edges, namely $(p, n)$ and $(n, q)$, and then by Proposition 3.6. we have $N_{s}(m, n)=\mathrm{W}_{\{(i, j)\}}$, as before. The other possibility is that, in the round when $n$ was first played, the two edges labeled by $\forall$ were $(q, n)$ and $(u, n)$, for some $u \in$ $N \backslash\{m, n, p, q\}$. Now consider $\{m, n, p, q, u\}$. Recall that $n$ is the most recently added node. Since $N_{s}(p, n)$ is red and labeled by $\exists$, it must be that $N_{s}(p, u)$ and $N_{s}(u, n)$ are both green, by Proposition 3.6. But then, by Proposition 3.6. again, $N_{s}(m, n)=\mathrm{W}_{\{(i, j)\}}$, as before. This proves the claim.

But then, since the two atoms $\alpha$ and $\beta$ used in $\forall$ 's current move have to satisfy $\alpha ; \beta \geq$ $N_{s}(m, n)$ and we are assuming that $\alpha$ is green and $\beta$ is yellow, it must be that $\alpha=g_{i}$ and $\beta=\mathrm{y}_{j}$. Then $\exists$ would have used Part 1 of her strategy, since there is a witness node $q \in N_{s}$, contrary to assumption. This proves that 3(a) and 3(c) cannot simultaneously apply.

Finally we check that 3(c) cannot apply simultaneously because of distinct nodes $q, q^{\prime} \in$ $N_{s}$ (see the second part of Figure 6). For contradiction suppose it did. Then $N_{s}(m, p)$, $N_{s}(m, q)$, and $N_{s}\left(m, q^{\prime}\right)$ are all green and $N_{s}(p, q), N_{s}(q, n), N_{s}\left(p, q^{\prime}\right)$, and $N_{s}\left(q^{\prime}, n\right)$ are all yellow. Consider the nodes $\left\{m, n, p, q, q^{\prime}\right\}$. It is easy to check that there are at least four nodes in this set (the only possible equality is $q=q^{\prime}$ ). By Proposition 3.6., the most recently added node is $n$, as all the other nodes are incident with at least three $\forall$-edges from this set. Proposition 3.6. also implies that $N_{s}(m, n)=\mathrm{W}_{\left\{(i, j),\left(i^{\prime}, j^{\prime}\right)\right\}}$ where $N_{s}(m, q)=\mathrm{g}_{i}, N_{s}(q, n)=\mathrm{y}_{j}, N_{s}\left(m, q^{\prime}\right)=\mathrm{g}_{i^{\prime}}$ and $N_{s}\left(q^{\prime}, n\right)=\mathrm{y}_{j^{\prime}}$. But then the current move $(m, n, x, y)$ would have been covered by Part 1 of her strategy (using either $q$ for $\alpha=\mathrm{g}_{i}$ and $\beta=\mathrm{y}_{j}$ or $q^{\prime}$ for $\alpha=\mathrm{g}_{i^{\prime}}$ and $\beta=\mathrm{y}_{j^{\prime}}$ as a witness), contrary to assumption.

Now we check that her strategy is well defined when she uses Parts 4 and 5 and chooses a red atom. If Part 5 applies, then by Definition 3.4., whether the red cluster is of Type 1 or Type $2, N_{s}(m, n)$ cannot be white, hence Part 4 cannot apply. If Part 5 applies, then it is well defined, since the red cluster containing $l$ and $p$ is unique, by Lemma 3.7.

Note that the use of Part 5 of $\exists$ 's strategy ensures that our inductive condition remains true in $N_{s+1}$. Having shown that $\exists$ 's strategy is well defined, we are now ready to prove that she can win the game.

LEMMA 3.9. If $6 \cdot 2^{t} \leq n$, then $\exists$ has a winning strategy in $\mathcal{G}_{t}\left(\mathfrak{A}_{n}\right)$.

Proof. It suffices to prove that $N_{s+1}$ is indeed a network. Triangles not involving the new node $l$ are consistent, since $N_{s}$ is a network. The triangle $(m, n, l)$ must be consistent, since 
$\forall$ is required to pick $\alpha$ and $\beta$ with $\alpha ; \beta \geq N_{s}(m, n)$. Primary triangles $(l, m, p)$ and $(l, n, p)$ for $p \in N_{s} \backslash\{m, n\}$ can easily be checked to be consistent, by inspecting each case of $\exists$ 's strategy. It remains to check the secondary triangles $(l, p, q)$, where $p, q \in N_{s} \backslash\{m, n\}$. From her strategy, $N_{s+1}(l, p)$ and $N_{s+1}(l, q)$ are white, black, or red. The only forbidden triples involving two atoms of these colors are (18) and (20).

To check forbidden triple (18), suppose that $N_{s+1}(l, p)=\mathrm{b}_{i}, N_{s+1}(l, q)=\mathrm{r}_{j}$ (some $i, j<n)$ and $N_{s}(p, q)=\mathrm{y}_{k}($ some $k<n)$. Then $\alpha, N_{s}(m, p)$ and $N_{s}(m, q)$ are all green (or symmetrically $\beta, N_{s}(n, p)$ and $N_{s}(n, q)$ are all green), otherwise $\exists$ would have chosen white instead of red and black. See Figure 7. Since $N_{s+1}(l, p)$ is black, $\exists$ must have used Part 3 of her strategy when labeling $(l, p)$. There are two possibilities which account for $N_{s+1}(l, q)$ being red, namely Parts 4 and 5 . So either $\beta$ and $N(n, q)$ are both yellow, or there is a yellow path from $l$ to $q$ within $\mathrm{G}_{N_{s+1}}(m)$. Either way $\beta$ is yellow, as shown in the figure. First suppose that $N_{s}(n, q)$ is yellow and $N_{s}(m, n)$ is not green (this covers the cases where $(l, q)$ was labeled using Part 4 of $\exists$ 's strategy, and also Part 5 when $l$ and $q$ belong to a red cluster of the first type). In this case, Part $3 \mathrm{c}$ applies when labeling $(l, p)$, so $\exists$ must have selected $N_{s+1}(l, p)=\mathrm{b}_{k}$, that is, $k=i$, so the triangle is consistent. Next suppose that there is a yellow path $\rho$ from $l$ to $q$ within $\mathrm{G}_{N_{s+1}}(m)$. Then, by appending $p$ to $\rho$, we see that there is a yellow path from $l$ to $p$ within $\mathrm{G}_{N_{s+1}}(m)$, so $l$ and $p$ belong to the same red cluster of $N_{s+1}$. This is contrary to our assumption that Part 3 applied when labeling $(l, p)$.

To check forbidden triple (20), suppose $N_{s+1}(l, p), N_{s+1}(l, q)$, and $N(p, q)$ are all red. Since $N_{s+1}(l, p)$ is red, it must have been selected by either Part 4 or 5 of $\exists$ 's strategy. In the former case, $N_{s}(m, n)=\mathrm{w}_{S}, \alpha$ and $N_{s}(m, p)$ are green, $\beta$ and $N_{s}(n, p)$ are yellow. Since $N_{s}(m, n)$ is white, $(l, q)$ must also have been labeled using Part 4 of $\exists$ 's strategy (Part 5 requires that $N_{s}(m, n)$ is not white), so $N_{s}(m, q)$ is also green and $N_{s}(n, q)$ is yellow. Consider the set $\{m, n, p, q\}$. By Proposition 3.6. there are two possibilities for the most recently added node. If it is either $m$ or $n$, then $\exists$ chose $N_{s}(m, n)=\mathbf{W}_{\left\{\left(i^{\prime}, j^{\prime}\right),\left(i^{*}, j^{*}\right)\right\}}$ where $N_{s}(m, p)=\mathrm{g}_{i^{\prime}}, N_{s}(p, n)=\mathrm{y}_{j^{\prime}}, N_{s}(m, q)=\mathrm{g}_{i^{*}}$, and $N_{s}(q, n)=\mathrm{y}_{j^{*}}$. If $\alpha=\mathrm{g}_{i}$ and $\beta=\mathrm{y}_{j}$, then, since $\alpha ; \beta \geq N_{s}(m, n)=\mathrm{w}_{\left\{\left(i^{\prime}, j^{\prime}\right),\left(i^{*}, j^{*}\right)\right\}}$, either $(i, j)=\left(i^{\prime}, j^{\prime}\right)$ or $(i, j)=\left(i^{*}, j^{*}\right)$. Either way, $\exists$ would have responded to the current move using Part 1 of her strategy, using either $p$ or $q$ as a witness, contrary to assumption. The other possibility is that the most recently added node of $\{m, n, p, q\}$ is either $p$ or $q$, but then $\exists$ would have chosen a white atom to label $N_{s}(p, q)$, by Proposition 3.6., contrary to our assumption that $N_{s}(p, q)$ is red.

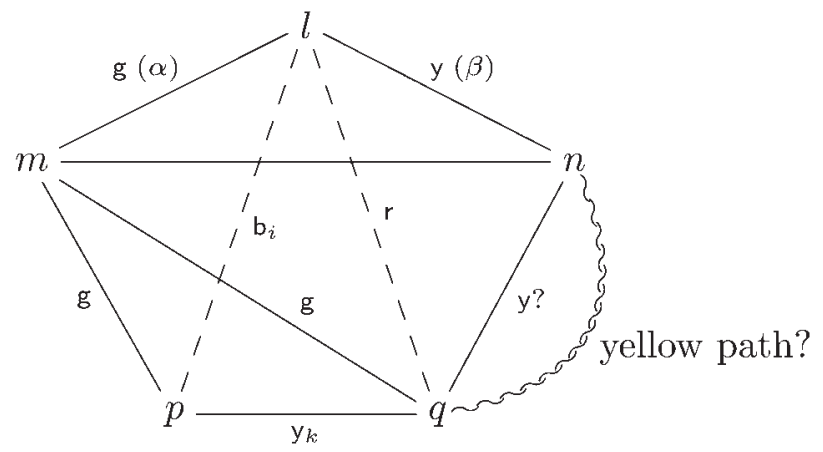

Fig. 7. Checking consistency. 
So we may suppose that both $(l, p)$ and $(l, q)$ were labeled using Part 5 of $\exists$ 's strategy. Thus $l$ and $p$ belong to a red cluster of $N_{s+1}$, and $l$ and $q$ belong to a red cluster of $N_{s+1}$. We claim that $l, p$, and $q$ all belong to the same red cluster of $N_{s+1}$. To prove this claim, note that each node in a red cluster is incident with at least one green edge and at least one yellow edge, so $\alpha$ must be green and $\beta$ must be yellow (or the other way round). If the red cluster containing $l$ and $p$ is of Type 1 , then $N_{s}(m, n)$ is neither white nor green, so the red cluster containing $l$ and $q$ is also of Type 1 . In this case, $\alpha, N_{s}(m, p)$ and $N_{s}(m, q)$ are green, and $\beta, N_{s}(n, p)$ and $N_{s}(n, q)$ are yellow, so $l, p, q \in \mathrm{G}_{N_{s+1}}(m) \cap \mathrm{Y}_{N_{s+1}}(n)$, that is, all three points belong to the same red cluster. If $l, p$ belong to a red cluster of Type 2 , then $N_{s}(m, n)$ must be green and there is a yellow path from $l$ to $p$ in $\mathrm{G}_{N_{s+1}}(m)$. Then the red cluster containing $l$ and $q$ must also be of Type 2, and there is a yellow path from $l$ to $q$ in $\mathrm{G}_{N_{s+1}}(m)$. Hence, $l, p$, and $q$ all belong to the same $\sim_{m}$-equivalence class of $\mathbf{G}_{N_{s+1}}(m)$, so again all three points belong to the same red cluster, as claimed.

But then $l, p$, and $q$ belong to a red cluster $X$ of $N_{s+1}$ with associated $(t-(s+1))$-good map $f^{+}: X \rightarrow(n+1)$, and the edges of the triangle $(l, p, q)$ have labels $\mathbf{r}_{|f(l)-f(p)|}$, $\mathbf{r}_{|f(p)-f(q)|}$, and $\mathbf{r}_{|f(l)-f(q)|}$, whence the triangle is consistent by (21).

LEMma 3.10. Any nonprincipal ultraproduct $\mathfrak{A}$ of $\left(\mathfrak{A}_{n}: n \in \omega\right)$ over $\omega$ is in RRA. Hence the ultraproduct $\mathfrak{D}$ of the $\{\cdot,+, \backslash\}$-reducts $\mathfrak{D}_{n}$ of $\mathfrak{A}_{n}$ is representable as well: $\mathfrak{D} \in$ $\mathrm{Q}^{c d}(\cdot,+, \backslash)$.

Proof. We have seen that $\exists$ has a winning strategy in $G_{t}\left(\mathfrak{A}_{n}\right)$, provided $6 \cdot 2^{t} \leq n$. A standard argument now shows that $\exists$ has a winning strategy in a countably long game over a nonprincipal ultraproduct $\mathfrak{A}$ of the $\mathfrak{A}_{n}$ s. The rough idea of $\exists$ 's winning strategy in $G_{\omega}(\mathfrak{A})$ is the following. For each $n$, let $t(n)=\left\lfloor\log _{2}\left(\frac{n}{6}\right)\right\rfloor$. Let $U$ be the nonprincipal ultrafilter used to define the ultraproduct. In the initial round of $G_{\omega}(\mathfrak{A}), \forall$ picks an atom $[a]$ of $\mathfrak{A}$. Here $a=\left(a_{0}, a_{1}, \ldots\right)$ for some sequence of elements $a_{i} \in \mathfrak{A}_{i}$, and $[a]$ denotes the equivalence class of $a$ (where equivalence is 'identical over a set of indices belonging to $U$ '). For a 'large' set $S_{0}$ of indices $n$ (i.e., $S_{0} \in U$ ) the element $a_{n}$ must be an atom of $\mathfrak{A}_{n}$. There are only finitely many values of $n$ for which $t(n)=0$, hence $S_{1}=\left\{n \in S_{0}: t(n)>0\right\} \in U$. For each $n \in S_{1}, \exists$ supposes that $\forall$ plays the atom $a_{n}$ in the initial round of $G_{t(n)}\left(\mathfrak{A}_{n}\right)$, and she responds using her winning strategy for $G_{t(n)}\left(\mathfrak{A}_{n}\right)$ with the $\mathfrak{A}_{n}$-network $N_{0}^{n}$. We may suppose that each of these networks has at most two nodes for a 'large' subset $S_{2} \subseteq S_{1}$, and that they all have the same set of nodes, that is, $\left\{N_{0}^{n}: n \in S_{2}\right\}$ all have the same nodes, $X$ say. For indices $n$ not in $S_{2}$ we may (re-)define $N_{0}^{n}$ as an arbitrary network with nodes $X$. We may define the 'ultraproduct' $N_{0}$ of the $N_{0}^{n}$ s as the network with nodes $X$ and for $x, y \in X$ we have $N_{0}(x, y)=\left[\left(N_{0}^{n}(x, y): n \in \omega\right)\right] . N_{0}$ is the initial network in the match of $G_{\omega}(\mathfrak{A})$. At the start of round $r>0$, suppose inductively that we have $\mathfrak{A}_{n}$-networks $N_{r-1}^{n}$ and that $N_{r-1}$ is the ultraproduct of the $N_{r-1}^{n} \mathrm{~s}$ in the above sense. We also suppose inductively that for 'many' indices $n$, the network $N_{r-1}^{n}$ occurs in a match of $G_{t(n)}\left(\mathfrak{A}_{n}\right)$ in which $\exists$ has been using her winning strategy. For his move, $\forall$ picks an edge $(x, y)$ of $N_{r-1}$ and atoms $[a],[b]$ of $\mathfrak{A}$ such that $[a] ;[b] \geq N_{r-1}(x, y)$. Let $a=\left(a_{0}, a_{1}, \ldots\right)$ and $b=\left(b_{0}, b_{1}, \ldots\right)$. For each $n>6 \cdot 2^{r}, \exists$ supposes that $\forall$ plays the edge $(x, y)$ and $a_{n}, b_{n} \in \operatorname{At}\left(\mathfrak{A}_{n}\right)$ in round $r$ of $G_{t(n)}\left(\mathfrak{A}_{n}\right)$. For 'many' values of $n$, this will be a legitimate move for $\forall$ in that game. For all of these games, provided $n>6 \cdot 2^{r}, \exists$ plays the network $N_{r}^{n}$ according to her winning strategy in that game - note that only finitely many strategies will have expired. For values of $n$ outside this 'large' set, we can let $N_{r}^{n}$ be an arbitrary network-this will not have any effect on the ultraproduct network $N_{r}$. In this way, $\exists$ 


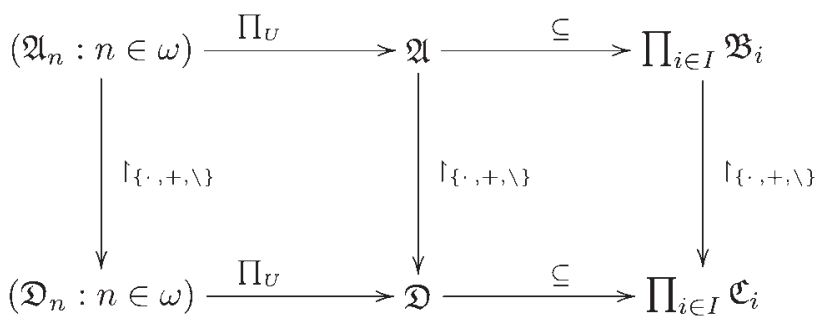

Fig. 8. Reducts and ultraproducts.

maintains the inductive hypotheses. Since $N_{r}$ is the ultraproduct of a sequence of networks $N_{r}^{n}$, it must be indeed a network of $\mathfrak{A}$, hence $\exists$ will not lose in round $r$. The details of this argument may be found in Hirsch \& Hodkinson (1997, Proposition 15), or for a more general argument see Hirsch \& Hodkinson (2002, Section 10.5.1).

Trivially, since $\exists$ has a winning strategy in $G_{\omega}(\mathfrak{A})$, she has a winning strategy in $G_{n}(\mathfrak{A})$ for all $n \in \omega$. By Proposition 3.3., the ultraproduct $\mathfrak{A} \in \mathrm{RRA}=$ SPRs. Hence there are $\mathfrak{B}_{i} \in \mathrm{Rs}$ for $i \in I$ such that $\mathfrak{A} \subseteq \prod_{i \in I} \mathfrak{B}_{i}$. Note that $\mathfrak{A}$ is commutative and dense, since each $\mathfrak{A}_{n}(n \in \omega)$ has these properties. Since every $\mathfrak{B}_{i}(i \in I)$ is a homomorphic image of $\mathfrak{A}$ (see Hirsch \& Hodkinson, 2002, Lemma 3.7.), each $\mathfrak{B}_{i}$ is commutative and dense as well. Let $\mathfrak{D}_{n}$ be the $\{\cdot,+, \backslash\}$-reduct of $\mathfrak{A}_{n}$, let $\mathfrak{D}$ be the ultraproduct of $\mathfrak{D}_{n}(n \in \omega)$ and $\mathfrak{C}_{i}$ be the $\{\cdot,+, \backslash\}$-reduct of $\mathfrak{B}_{i}$ for $i \in I$, that is, $\mathfrak{C}_{i} \in \mathrm{R}^{c d}(\cdot,+, \backslash)$ (see Figure 8). Then $\mathfrak{D}$ is the $\{+, \cdot, \backslash\}$-reduct of $\mathfrak{A}$, and hence $\mathfrak{D} \subseteq \prod_{i \in I} \mathfrak{C}_{i}$. That is, $\mathfrak{D} \in \operatorname{SPR}^{c d}(\cdot,+, \backslash) \subseteq$ $\mathrm{Q}^{c d}(\cdot,+, \backslash)$ as desired.

\$4. Conclusion. The variety and quasivariety generated by $R(\cdot, ;, \backslash, /)$ coincide, and it is a finitely axiomatizable class (Andréka \& Mikulás, 1994). By adding the axioms of commutativity and density, we get finite axiomatization for the (quasi)variety $\mathrm{Q}^{c d}(\cdot, ;, \backslash$ ) generated by $\mathrm{R}^{c d}(\cdot, ;, \backslash)$. We have seen above that we lose finite axiomatizability (with or without commutativity and/or density) if we include + .

Problem 4.1. Are the (quasi)equational theories of (commutative and/or dense subclasses of $) \mathrm{R}(+, \backslash, /)$ and $\mathrm{R}(+, \backslash, /, ;)$ finitely based?

We showed in Theorem 2.3. that both the equational and quasiequational theories of $\mathrm{R}(\cdot,+, \backslash, /)$ are not finitely axiomatizable, but we left the question whether $\mathrm{Q}(\cdot,+, \backslash, /)$ and $\mathrm{V}(\cdot,+, \backslash, /)$ coincide open. We can formulate the problem in a more general setting.

PROBLEM 4.2. For which similarity types $\tau$ of residuated algebras $\mathrm{Q}(\tau)=\mathrm{V}(\tau)$ ?

In connection with this problem we note that $\mathrm{Q}(\cdot, ;, \backslash, /)=\mathrm{V}(\cdot, ;, \backslash, /$ ) (cf. Pratt, 1990; Mikulás, submitted), and it would be interesting to see a more general method for translating quasiequations to equivalent equations using the residuals.

A challenging line of research is to find finite quasiequational axiomatizations for nonfinitely axiomatizable varieties.

PROBLEM 4.3. For a nonfinitely axiomatizable variety $\mathrm{V}$, find a finitely axiomatizable quasivariety $\mathrm{Q}$ such that $\mathrm{V}$ is the variety generated by $\mathrm{Q}$.

Translating such a result to logic would provide us with a weakly sound and complete Hilbert-style calculus. We note that the axiomatization given in Kozen (1994a) for Kleene algebras is such an example. 
Finally we note that Theorem 2.3. can be strengthened so that $\tau$ includes the reflexivetransitive closure * operation as well. Indeed, in $\mathfrak{A}_{n}, a ; a ; a=1$ for every nonidentity atom $a$. Thus we can define

$$
x^{*}= \begin{cases}1^{\prime} & x \in\left\{0,1^{\prime}\right\} \\ 1 & \text { otherwise }\end{cases}
$$

for every $x \in A_{n}$. Thus $x^{*}$ can be defined as $(x ; x ; x)+1^{\prime}$ in $\mathfrak{A}_{n}$, hence in the ultraproduct $\mathfrak{A}$. As before, the $\tau$-reduct of $\mathfrak{A}_{n}$ is not representable. By the definition of * above, we have that the ultraproduct is representable even if * $\in \tau$. In particular, we have nonfinite axiomatizability for representable residuated Kleene lattices, a.k.a. action lattices (Kozen, 1994b).

COROLLARY 4.4. The quasivariety and variety generated by $\mathrm{R}\left(\cdot,+,, ;, \backslash, /,{ }^{*}, 0,1^{\prime}\right)$ are not finitely axiomatizable.

§5. Acknowledgement. Thanks are due to the two anonymous referees for their useful comments and suggestions.

\section{BIBLIOGRAPHY}

Anderson, A. R., \& Belnap, N. D. (1975). Entailment. The Logic of Relevance and Necessity. Vol. I. Princeton, NJ: Princeton University Press.

Anderson, A. R., Belnap, N. D., \& Dunn, J. M. (1992). Entailment. The Logic of Relevance and Necessity. Vol. II. Princeton, NJ: Princeton University Press.

Andréka, H. (1988). On the representation problem of distributive semilattice-ordered semigroups. Preprint, Mathematical Institute of the Hungarian Academy of Sciences. Abstracted in Abstracts of the American Mathematical Society, 10(2), 174 (March 1989).

Andréka, H. (1991). Representation of distributive lattice-ordered semigroups with binary relations. Algebra Universalis, 28, 12-25.

Andréka, H., \& Mikulás, Sz. (1994). Lambek calculus and its relational semantics: Completeness and incompleteness. Journal of Logic, Language and Information, 3, 137.

Andréka, H., \& Mikulás, Sz. (accepted for publication). Axiomatizability of positive algebras of binary relations.

van Benthem, J. (1996). Exploring Logical Dynamics. Stanford, CA: CSLI Publications.

Bimbó, K., Dunn, J. M., \& Maddux, R. D. (2009). Relevance logic and relation algebras. Review of Symbolic Logic, 2(1), 102-131.

Bredikhin, D. A., \& Schein, B. M. (1978). Representation of ordered semigroups and lattices by binary relations. Colloquium Mathematicum, 39, 1-12.

Došen, K. (1992). A brief survey of frames for the Lambek calculus. Zeitschrift für Mathematische Logik und Grundlagen der Mathematik, 38, 179-187.

Dunn, J. M. (1966). The Algebra of Intensional Logics. PhD Dissertation, University of Pittsburgh.

Dunn, J. M. (1982). Relational representation of quasi-Boolean algebras. Notre Dame Journal of Formal Logic, 23, 353-357.

Dunn, J. M. (1993) Partial gaggles. In Došen, K. and Schroeder-Heister, P. editors. Substructural Logics. Oxford, UK: Clarendon, pp. 63-109.

Gehrke, M., Dunn, M., \& Palmigiano, A. (2005). Canonical extensions and relational completeness of some substructural logics. Journal of Symbolic Logic, 70(3), 713-740. 
Hirsch, R. (1995). Completely representable relation algebras. Bulletin of the IGPL, 3(1), 77-92.

Hirsch, R. (2005). The class of representable ordered monoids has a recursively enumerable, universal axiomatisation but it is not finitely axiomatisable. Logic Journal of the IGPL, 13, 159-171.

Hirsch, R., \& Hodkinson, I. (1997). Complete representations in algebraic logic. Journal of Symbolic Logic, 62, 816-847.

Hirsch, R., \& Hodkinson, I. (2000). Relation algebras with $n$-dimensional relational bases. Annals of Pure and Applied Logic, 101, 227-274.

Hirsch, R., \& Hodkinson, I. (2001a). Relation algebras from cylindric algebras, II. Annals of Pure and Applied Logic, 112, 267-297.

Hirsch, R., \& Hodkinson, I. (2001b) Representability is not decidable for finite relation algebras. Transactions of the American Mathematical Society, 353, 1403-1425.

Hirsch, R., \& Hodkinson, I. (2002). Relation Algebras by Games. Amsterdam, The Netherlands: North-Holland.

Hirsch, R., \& Hodkinson, I. (2009). Strongly representable atom structures of cylindric algebras. Journal of Symbolic Logic, 74, 811-828.

Hirsch, R., \& Mikulás, Sz. (2007). Representable semilattice-ordered monoids. Algebra Universalis, 57, 333-370.

Hodkinson, I. (1997) Atom structures of cylindric algebras and relation algebras. Annals of Pure and Applied Logic, 89, 117-148.

Hodkinson, I., \& Mikulás, Sz. (2000). Axiomatizability of reducts of algebras of relations. Algebra Universalis, 43, 127-156.

Jónsson, B. \& Tarski, A. (1952). Boolean algebras with operators, part II. American Journal of Mathematics, 74, 127-162.

Kowalski, T. (2007). Weakly associative relation algebras hold the key to the universe. Bulletin of the Section of Logic, 36, 145-157.

Kozen, D. (1994a). A completeness theorem for Kleene algebras and the algebra of regular events. Information and Computation, 110, 366-390.

Kozen, D. (1994b). On action algebras. In van Eijck, J. and Visser, A. editors. Logic and Information Flow. Cambridge, MA: MIT Press, pp. 78-88.

Maddux, R. D. (2006). Relation Algebras. Amsterdam, The Netherlands: North-Holland.

Maddux, R. D. (2007). Relevance logic and the calculus of relations. Paper presented at International Conference on Order, Algebra and Logics. Department of Mathematics, Vanderbilt University, June 12-16, 2007. Abstract available from: http://www.math.vanderbilt.edu/ oal2007/submissions/submission_10.pdf. More detailed notes available from: http://www.math.iastate.edu/maddux/talk.pdf.

Maddux, R. D. (2010). Relevance logic and the calculus of relations. Review of Symbolic Logic, 3(01), 41-70.

Meyer, R. K., \& Routley, R. (1972). Algebraic analysis of entailment I. Logique et Analyse, 15, 407-428.

Mikulás, Sz. (2004). Axiomatizability of algebras of binary relations. In Löwe, B., Piwinger, B., and Räsch, T. editors. Classical and New Paradigms of Computation and Their Complexity Hierarchies. Dordrecht, The Netherlands: Kluwer Academic Publishers, pp. 187-205.

Mikulás, Sz. (2009). Algebras of relations and relevance logic. Journal of Logic and Computation, 19, 305-321.

Mikulás, Sz. (Submitted). On representable ordered residuated semigroups. 
Monk, J. (1964). On representable relation algebras. Michigan Mathematics Journal, 11, 207-210.

Pratt, V. (1990). Action logic and pure induction. In van Eijck, J. editor. Logics in AI: European Workshop JELIA '90. Heidelberg, Germany: Springer-Verlag, pp. 97-120.

Routley, R., \& Meyer, R. K. (1973). The semantics of entailment (I). In Leblanc, H. editor. Truth, Syntax and Modality. Amsterdam, The Netherlands: North-Holland, pp. 199-243.

Schein, B. M. (1991). Representation of subreducts of Tarski relation algebras. In Andréka, H., Monk, J. D., and Németi, I. editors. Algebraic Logic. Amsterdam, The Netherlands: North-Holland, pp. 621-635.

Tarski, A. (1954). Contributions to the theory of models, I, II. Proceedings Koninklijke Nederlandse Akadedemie van Wetenschappen, 57, 572-581 (= Indagationes Mathematicae, 16, 582-588).

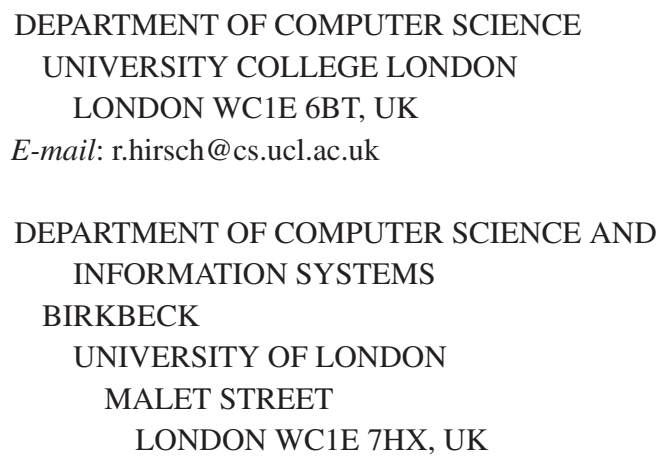

E-mail: r.hirsch@cs.ucl.ac.uk

E-mail: szabolcs@dcs.bbk.ac.uk 